\title{
Données actuelles sur les Acariens Pyroglyphides des poussières de maison
}

\author{
par A. PENAUD, J. NOURRIT, P. AUTRAN, P. TIMON-DAVID et R.-M. NICOLI \\ Laboratoire de Parasitologie, Faculté de Médecine, Secteur Nord, \\ boulevard P.-Dramard, F 13015 Marseille
}

\section{Résumé}

Les auteurs rapportent le résultat de leurs recherches des Acariens Pyroglyphides dans les échantillons de poussières domestiques.

Ils rappellent leur technique de recherche, les principales caractéristiques morphologiques, biologiques et écologiques des Acariens rencontrés; ils évoquent par ailleurs l'allergénicité de ces «parasites » et les mesures thérapeutiques et prophylactiques qui pourraient leur être opposées.

\section{Summary}

The authors report the result of their research on Pyroglyphid mites in house-dust samples.

They bring to mind their research technics, the principal morphological, biological and ecological characteristics of the Acari discovered. Moreover they recall the allergenicity of these «parasites » and the therapeutic and prophylactic measures which could be used against them.

Les Acariens Pyroglyphides des poussières de maison ont pris une importance toute nouvelle en pathologie humaine, depuis que R. Voorhorst et F. Th. M. Spieksma

Nous voudrions adresser nos vifs remerciements à $\mathbf{M}$. le Professeur A. Fain et à M. le Docteur A. M. Cunnington qui nous ont beaucoup aidé au début de nos recherches. 
ont montré que l'un de ces acariens, appartenant au genre Dermatophagoïdes, était responsable des phénomènes allergiques déclenchés par la poussière de maison $[30,31$, $32,33,35,36,37,39]$. Par la suite, cette découverte a été confirmée par les auteurs anglais [8,9,17,22,26], belges [12, 13,14], suisses [27], japonais [18, 19, 20, 21] et américains [40].

A la demande du $\mathrm{Pr}^{\mathrm{r}} \mathrm{J}$. Charpin (professeur de clinique pneumo-phtisiologique à la Faculté de Médecine de Marseille), nous avons entrepris la recherche de ces «parasites » dans les poussières de maisons habitées par des malades présentant des manifestations d'allergie respiratoire (asthme bronchique ou coryza spasmodique); les poussières que nous avons analysées provenaient essentiellement du sud-est de la France.

Après avoir rapporté dans des publications antérieures $[6,24]$ nos premiers résultats, nous les complétons ici par une étude plus approfondie et portant sur un plus grand nombre d'échantillons.

\section{I. - Historique}

R. Voorhorst, M ${ }^{\text {me }}$ M. I. A. Spieksma-Boezeman et F. Th. M. Spieksma, de l'Institut allergologique de Leiden, en Hollande, montrent en 1964 [36] que l'asthme bronchique est dû à l'inhalation d'acariens du genre Dermatophagoïdes présents dans les poussières de maison d'habitation et non par la poussière elle-même comme on le croyait jusqu'alors; en 1965, Voorhorst montre que les produits d'excrétion ou de sécrétion de ces acariens constituent des allergènes aussi actifs que les acariens euxmêmes.

En 1966, A. Fain [12, 13], de l'Institut Prince-Léopold d'Anvers, qui à la demande des auteurs hollandais avait identifié spécifiquement le Dermatophagoïdes responsable de ces accidents allergiques comme étant le Dermatophagoïdes pteronyssinus, redécrit ce dernier. En effet, le $D$. pteronyssinus (Trouessart), qui avait été la première fois décrit en 1897 par Berlèse sous le nom de Mealia pteronyssina, n'avait plus été retrouvé depuis cette date, les spécimens de cette espèce étant perdus. Fain, grâce à des paratypes de la préparation originale de Trouessart, conservés à l'acarothèque de Berlèse à Florence, redécrit $D$. pteronyssinus et désigne un lectotype. Il entreprend par la suite de son côté un examen des poussières de maison dans différentes villes de Belgique, puis étend ses recherches à Florence, Léopoldville et différentes villes du Brésil.

Il constate presque chaque fois que $D$. pteronyssinus est présent dans les poussières domestiques, ce qui montre son caractère cosmopolite. En dehors de D. pteronyssinus et avec une fréquence nettement moins grande que ce dernier, d'autres espèces de Dermatophagoïdes, en particulier $D$. farinae, et d'autres acariens apparentés, tel Euroglyphus maynei, sont trouvés aussi bien par Voorhorst et Spieksma que par Fain. 
En 1967 et 1968, K. Maunsell, D. G. Wraith et A. M. Cunnington [17] aboutissent en Angleterre aux mêmes conclusions. Cunnington met en évidence le rôle de niche écologique joué par le matelas. En 1968, J. Pepys et ses collaborateurs [25, 26] étudient à Londres l'importance clinique relative des extraits de $D$. pteronyssinus et D. culinae $(=D$. farinae $)$ dans l'allergie aux poussières de maison. Il constate qu'il y a un rapport certain, quoique non absolu, entre la notion clinique d'allergie aux poussières de maison et les réactions cutanées ou inhalatoires obtenues avec des extraits de $D$. culinae et $D$. pteronyssinus. Il montre en outre que le fractionnement par le sulfate d'ammonium des extraits de $D$. culinae fournit une fraction \&protéique » qui en «prick test » donne une réaction beaucoup plus intense que celle obtenue avec la fraction polysaccharidique du même $D$. culinae ou avec les extraits de $D$. pteronyssinus ou de poussière de maison.

Le rôle primordial du Dermatophagoïdes dans l'allergie aux poussières de maison a été mis en évidence aussi au Japon par T. Miyamoto et ses collaborateurs [18] qui publient les résultats de leurs travaux en 1968, et également aux U.S.A. par G.W Wharton [40] qui apporte les résultats de ses recherches en 1970. Wharton constate que $D$. farinae est beaucoup plus répandu que D. pteronyssinus aux U.S.A., alors que c'est le contraire en Hollande, en Belgique, en Angleterre et au Japon.

\section{II. - La famille des Pyroglyphidae}

La famille des Pyroglyphidae (1) fut érigée par Cunliffe en 1958. Fain fit des travaux importants sur la systématique de cette famille; entre autres, il fit entrer dans la famille des Pyroglyphidae les genres Dermatophagoïdes (2) et Sturnophagoüdes en 1967, puis le genre Malayoglyphus en 1969. Actuellement, la famille des Pyroglyphidae comprend quinze espèces dont huit se rencontrent dans la poussière de maison d'habitation [5] : il s'agit de Dermatophagoüdes pteronyssinus, Dermatophagoüdes farinae, Dermatophagoïdes chelidonis, Dermatophagoïdes evansi, Euroglyphus maynei, Euroglyphus longior, Sturnophagoïdes brasiliensis et Malayoglyphus intermedius.

Pour l'instant, la position systématique de cette famille s'établit schématiquement de la façon suivante:

(1) La famille Pyroglyphidae a été érigée par Cunliffe en 1958 pour un Acarien qui avait été découvert dans le nid d'un Rongeur et représentait une espèce et un genre nouveaux (Pyroglyphus morlani).

(2) Le genre Dermatophagoïdes a été créé par Bogdanov en 1864, pour une nouvelle espèce, D. Scheremetewsky qui avait été récoltée sur la peau de divers galeux à Moscou, tous porteurs de Sarcoptes scabiei. Ce genre semble avoir complètement échappé au grand acarologue Berlèse quand il décrivit l'Espèce pteronyssinus sous le nom du genre Mealia ainsi d'ailleurs qu'à d'autres acarologues connus tels que Trouessart, Canestrini..., les travaux de Bogdanov se perdirent probablement lors de la Révolution russe. Le genre Dermatophagoïdes tomba dans l'oubli jusqu'à ces dernières années. C'est en 1952 que Baker et Wharton firent tomber le genre Mealia en synonymie avec Dermatophagoïdes. La systématique du genre Dermatophagoïdes n'est peut-être pas encore définitivement établie. Le genre Dermatophagoïdes a longtemps fait partie de la famille des Epidermoptidae, mais Fain le transféra en 1963 dans la famille des Psoroptidae, et plus récemment en 1967 dans celle des Pyroglyphidae. Fain considère les représentants de ce genre comme étant les formes intermédiaires naturelles entre les Acariens libres et les Acariens parasites et dès lors ils sont selon lui les ancêtres de tous les Acariens qui parasitent les oiseaux et les mammifères. 


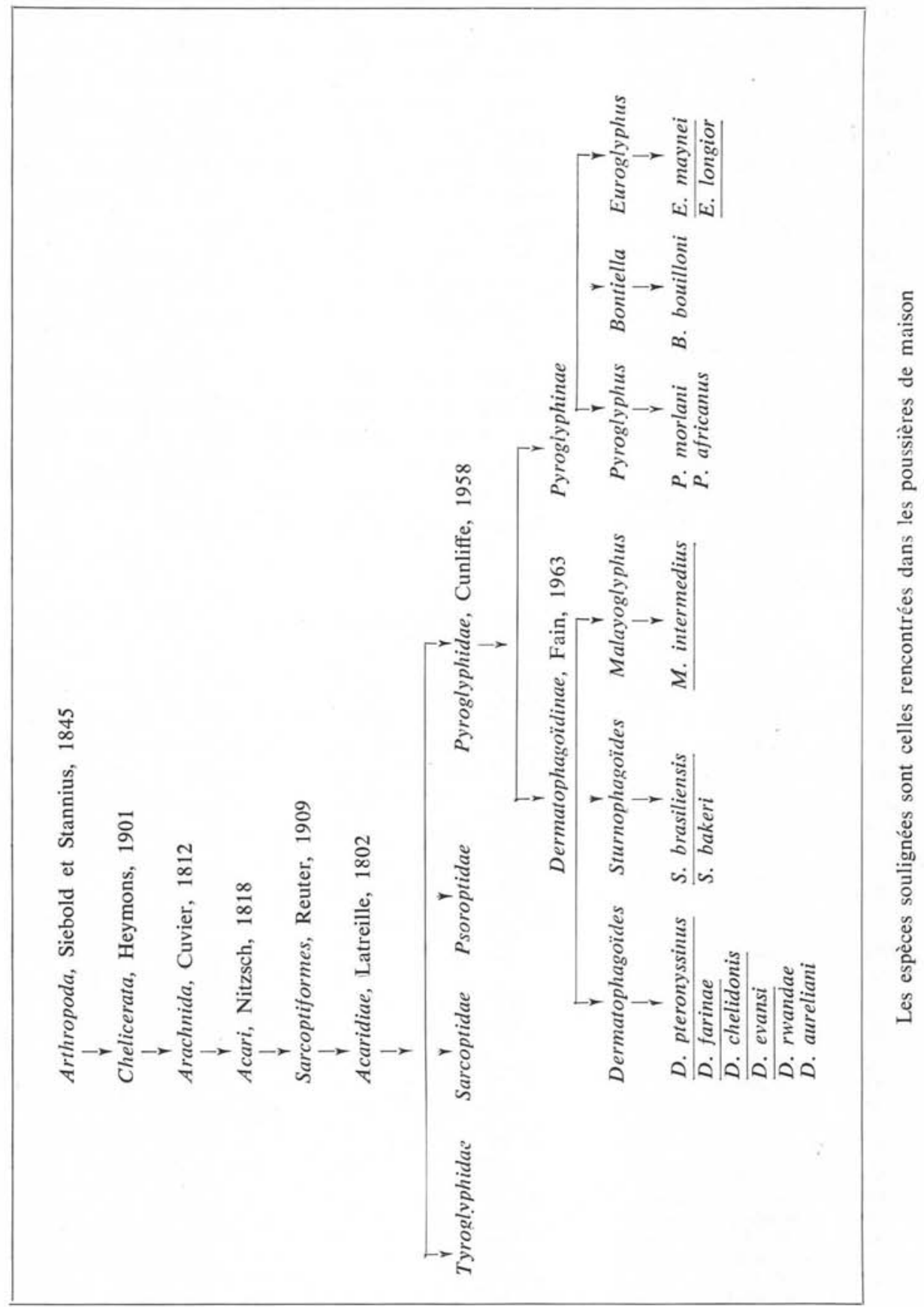


Notons que tous ces Acariens Pyroglyphides sont désignés sous le terme général de « mites» (3) par les auteurs anglo-saxons.

\section{III. - Morphologie}

Elle a été particulièrement bien étudiée par Fain $[10,11,12,13,14]$ qui a donné de tous les Acariens Pyroglyphides et des genres Dermatophagoïdes et Euroglyphus, en particulier, une description complète assortie de figures faisant autorité et servant de références de base pour les parasitologues. Nous nous sommes donc inspirés de ses travaux et nous rappelons les principales caractéristiques de ces Acariens.

\section{$1^{\circ}$ Le genre Dermatophagoüdes}

Les Dermatophagö̈des ont, à l'état adulte, quatre paires de pattes et sont pourvus d'appendices buccaux (chélicères et pédipalpes). Ils mesurent environ 300 à $400 \mu$ de long sur 200 à $250 \mu$ de large (4). Corps ovalaire dans les deux sexes, relativement court (par suite de la fusion du céphalothorax et de l'abdomen) et présentant un bord postérieur arrondi. Cuticule molle très mince, transparente et très finement striée sur tout le corps, sauf au niveau des écussons dorsaux. Face dorsale avec des écussons peu sclérifiés: l'écusson propodosomal existe dans les deux sexes. L'écusson hystérosomal est constant chez le mâle, absent chez la femelle. Sillon séjugal peu marqué ou absent. Absence d'apophyses rétrogrades sur le corps, les pattes ou le gnathosoma.

Pattes fines, relativement longues à cinq articles. Pattes IV chez la femelle égales aux pattes III ou plus courtes ou plus longues que celles-ci. Pattes III du mâle nettement plus fortes et plus longues que les pattes IV. Tarses I dans les deux sexes sans ongle ou avec un ou deux ongles plus ou moins fortement développés. Dans les deux sexes, le tarse I renferme un organe sensoriel spécial (le famulus). Tarses II avec ou sans ongle.

Epimères $\mathrm{I}$, bien séparés chez la femelle, séparés ou soudés en $\mathrm{V}$ ou en $\mathrm{Y}$ chez le mâle. Une ventouse contenant une griffe rudimentaire et droite est présente à toutes les pattes. Glande à huile présente. Epigynium chez la femelle, formant un arc plus ou moins fortement courbé, bien séparé des épimères $\mathrm{I}$; chez le mâle, les ventouses adanales sont bien développées. Vestiges des ventouses génitales présents. Présence de solenidions. Présence de poils sur l'idiosoma et sur les pattes. Dimorphisme sexuel très prononcé.

Telle est la description du genre Dermatophagoïdes Bogdanov. Les différentes espèces du genre présentent quelques variantes les unes par rapport aux autres.

(3) En Anglais : mite $=$ Acarien .

En Français : mite $=$ teigne (lépidoptère).

(4) Notons que le poids de ces Acariens est connu; Larson a évalué le poids moyen de D. farinae femelie adulte en culture à $16 \mu \mathrm{g}$ environ (à $25^{\circ} \mathrm{C}$ et à $75 \%$ d'humidité) [5]. 
Nous parlerons des variantes principales différenciant les quatre espèces de Dermatophagö̈des rencontrées dans les poussières de maison, en insistant surtout sur les deux plus importantes: D. pteronyssinus et $D$. farinae.

D. pteronyssinus Trouessart, 1897 (fig. 1, 2, 11, 12, 13, 14).

- Chez la femelle :

- La bursa copulatrix est très caractéristique. Elle est de calibre uniforme débouchant en arrière dans un petit vestibule et en avant dans une spermathèque dont seule la base bien visible est sclérifiée et dont la forme rappelle d'après son orientation soit une cupule soit une rosace.

- Epigynium cintré.

- Pattes III légèrement plus longues que pattes IV.

- Une partie des stries de la partie médiane de la face dorsale est orientée longitudinalement.

- Chez le mâle:

- Pattes I et II d'égale épaisseur.

- Ecusson hystérosomal dorsal plus long que large.

- Tarse I avec un seul ongle apical, tarse II sans ongle apical.

D. farinae Hughes, 1961 (= D. culinae De Léon, 1963) (fig. 3, 4, 5).

- Chez la femelle :

- L'orifice externe de la bursa copulatrix s'ouvre ventralement à droite ou à gauche de l'anus. Il est en forme de fente et débouche dans une petite poche conique à parois chitineuses et d'aspect ponctué. Au-delà de cette poche le canal de la bursa est très fin et de calibre uniforme, son embouchure interne est dépourvue de structure chitineuse et la spermathèque est complètement invisible.

- Epigynium beaucoup moins courbé que chez D. pteronyssinus.

- Pattes III plus courtes que pattes IV.

- Toutes les stries de la partie médiane de la face dorsale sont orientées transversalement. La striation est plus serrée que chez $D$. pteronyssinus.

- Forme de l'idiosoma largement ovalaire (la largeur maxima du corps se situe à la partie moyenne alors qu'elle se situe à la partie antérieure chez toutes les autres espèces).

- Chez le mâle:

- Pattes I beaucoup plus épaisses que les pattes II.

- Ecusson hystérosomal dorsal plus large que long.

- Epimères I très souvent contigus ou fusionnés en $\mathrm{V}$ ou surtout en $\mathrm{Y}$ avec un sternum plus ou moins long.

- Tarse III sans épines dans sa partie moyenne.

D. chelidonis Hull, 1931 (= D. passericola Fain, 1964).

Espèce qui présente la plus forte inégalité entre les pattes III et IV. 
- Chez la femelle:

- Pattes III beaucoup plus longues que pattes IV (alors que chez D. pteronyssinus et chez $D$. evansi, elles ne sont que légèrement plus longues et chez $D$. farinae, elles sont plus courtes).

- Pattes III plus épaisses que pattes IV (alors qu'elles sont d'égale épaisseur chez D. pteronyssinus, D. farinae et $D$. evansi).

- Epigynium relativement peu courbé.

- Canal de la bursa copulatrix très étroit, sans dilatations ni vestibule et dépourvu de structure chitineuse autour de son orifice interne. La bursa s'ouvre directement à l'extérieur au sommet d'une petite papille saillante située sur le bord postérieur du corps. La spermathèque est invisible.

- Chez le mâle:

- Pattes III deux fois plus longues que pattes IV (alors qu'elles ne le sont environ qu'une fois et demie chez D. pteronyssinus, D. farinae et $D$. evansi).

- Ecusson hystérosomal plus large que long.

- Cadre chitineux de la région anale bordé du côté interne par de très petites dents.

- Tarse III portant deux épines dans sa partie moyenne.

D. evansi Fain, Hughes et Johnston.

Se rapproche de $D$. pteronyssinus, mais s'en distingue par les caractères suivants :

- Chez la femelle:

- Epigynium plus fortement courbé et plus long que chez D. pteronyssinus.

- Le canal de la bursa copulatrix n'a pas de calibre uniforme comme chez D. pteronyssinus mais sa moitié postérieure est beaucoup plus dilatée que sa moitié antérieure. La partie postérieure débouche à l'extérieur par un orifice en entonnoir sans l'intermédiaire d'un vestibule. La partie antérieure s'abouche avec une spermathèque en grande partie sclérifiée et nettement plus longue que large.

- Chez le mâle:

- Ecusson hystérosomal dorsal plus long que chez $D$. pteronyssinus et il est plus fortement retréci en avant.

- Inégalité entre les pattes III et IV plus marquée que chez D. pteronyssinus.

- Cadre périanal plus grand que chez D. pteronyssinus.

- Tarses I et II respectivement terminés par deux et par un ongles.

\section{$2^{\circ}$ Le genre Euroglyphus}

Euroglyphus maynei Cooreman, 1950 (fig. 6, 7, 8).

Il ressemble au Dermatophagoïdes, mais il s'en différencie schématiquement par les caractères suivants : 
- Chez la femelle:

- Epaisseur des plis cuticulaires.

- Cuticule uniformément indurée (mais seulement légèrement sclérifiée, ponctuée en dehors des zones habituellement sclérifiées).

- Extrémité antérieure de l'idiosoma en coin.

- Existence d'un épistome bifide (pas d'épistome chez le Dermatophagoïdes).

- Région médiane de l'hystérosoma avec une large bande rectangulaire sclénifiée, ponctuée.

- Dépression légère au niveau de l'extrémité postérieure du corps (plus nette chez le mâle).

- Pattes III plus courtes que pattes IV (comme D. farinae, mais à l'inverse des autres Dermatophagoïdes).

- Grand écartement des Epimères I.

- Epigynium peu visible.

- Lèvre postérieure de la vulve sclérifiée, ponctuée.

- Papille génitale ovoïde, très sclérifiée, opaque et rougeâtre (débouchant latéralement et à proximité de l'extrémité postérieure de l'anus).

- Embouchure interne de la bursa copulatrix petite et très peu sclérifiée.

- Chaetotaxie nettement réduite.

- Chez le mâle:

- Epaisseur des plis cuticulaires.

- Cuticule uniformément indurée.

- Extrémité antérieure de l'idiosoma en coin.

- Existence d'un épistome, mais moins distinctement découpé que chez la femelle (pas d'épistome chez le Dermatophagoïdes).

- Hysterosoma avec un écusson ovalaire bien développé vers l'arrière du corps.

- Dépression très nette au niveau de l'extrémité postérieure du corps (le bord postérieur du corps est nettement découpé sur une bonne longueur mais sans former de véritables lobes).

- Pattes III non anormalement hypertrophiées comme chez le Dermatophagoïdes. Elles sont légèrement plus longues et guère plus épaisses que les pattes IV.

- Grand écartement des Epimères I.

- Chaetotaxie nettement réduite.

Euroglyphus longior Trouessart, 1897.

Il ressemble à l'Euroglyphus maynei, mais il s'en différencie schématiquement par les caractères suivants :

- Chez la femelle:

- Stries et plis plus nombreux.

- Ecusson propodosomal avec de nombreuses stries longitudinales très superficielles. 
- Hystérosoma avec seulement une petite zone triangulaire ponctuée située dans la partie postérieure du corps.

- Epistome étroit mais long et nettement bifide.

- Lèvre vulvaire postérieure très développée recouvrant complètement la fente vulvaire et la moitié interne des lèvres latérales (cette lèvre a une forme triangulaire et son angle antérieur est arrondi et entier).

- Pattes postérieures très fines.

- Chaetotaxie non réduite.

- Chez le mâle:

- Stries et plis moins nombreux.

- Epistome relativement long, se retrécissant fortement vers l'avant et fourchu apicalement.

- Partie postérieure du corps rétrécie et terminée par deux petits lobes.

- Finesse de toutes les pattes.

- Brièveté des Epimères I.

- Chaetotaxie non réduite.

Au terme de cette étude morphologique des Acariens Pyroglyphides, il convient de remarquer que les trois espèces essentielles à reconnaître, en raison de leur grande fréquence en Europe et de leur rôle pathogène, sont le Dermatophagoïdes pteronyssinus, le Dermatophagoïdes farinae et l'Euroglyphus maynei.

Nous n'avons pas décrit les genres Sturnophagoïdes et Malayoglyphus, car nous ne les avons jamais vus et, à notre connaissance, ils n'ont jamais été trouvés sur notre continent.

\section{IV. - Technique de recherche des Acariens Pyroglyphides dans les poussières de maison}

\section{$1^{\circ}$ Conditions de prélèvement}

- Les poussières de maison doivent être prélevées à la surface du matelas avec de préférence une brosse plutôt qu'un aspirateur (5). Brosser de préférence au niveau des contours et des nœuds du matelas.

- Prélever si possible $1 \mathrm{~g}$ de poussière. C'était le minimum que nous exigions au début de nos recherches. En fait, avec notre technique actuelle, nous pouvons maintenant examiner valablement des échantillons de quantité nettement plus réduite (de l'ordre de $50 \mathrm{mg}$ ).

(5) La poussière recueillie par aspirateur est trop fine ; c'est la raison pour laquelle Cunnington préconise le prélèvement par brossage, les résultats obtenus de cette manière étant bien meilleurs; c'est ce que nous avons également constaté nous-mêmes. 


\begin{tabular}{|c|c|c|c|c|c|c|}
\hline \multirow{3}{*}{$\begin{array}{l}\text { striation cuti- } \\
\text { culaire chez la } \\
\text { femelle }\end{array}$} & Dermatophagordes & pteronyssinus & permatophagoides & farinae & permatophagoides & chelidonis \\
\hline & $\mathrm{O}^{7}$ & ㅇ & $o^{\prime \prime}$ & 9 & $\sigma^{7}$ & Q \\
\hline & & $\begin{array}{l}\text { Une partie de } \\
\text { la face dorsa- } \\
\text { le mediane est } \\
\text { striee longitu- } \\
\text { dinalement }\end{array}$ & & $\begin{array}{l}\text { Partie dorsale } \\
\text { mediane entiere- } \\
\text { ment striee tr- } \\
\text { ansversalement } \\
\text { de maniere plus } \\
\text { serré }\end{array}$ & & $\begin{array}{l}\text { Une partie de Ia } \\
\text { face dorsale mé- } \\
\text { lane est striée } \\
\text { longitudinalement }\end{array}$ \\
\hline $\begin{array}{l}\text { Bursa } \\
\text { copulatrix }\end{array}$ & & $\begin{array}{l}\text { Calibre unifor- } \\
\text { me debouchant ea } \\
\text { arriere dans un } \\
\text { petit vestibule } \\
\text { et en avant dans } \\
\text { une spermatheque } \\
\text { dont seule la } \\
\text { base est scléri- } \\
\text { fiée et dont la } \\
\text { forme rappelle } \\
\text { une cupule ou } \\
\text { une rosace }\end{array}$ & & $\begin{array}{l}\text { Orifice externe } \\
\text { en forme de fen- } \\
\text { te debouchant } \\
\text { dans une poche } \\
\text { conique a paroi } \\
\text { chitineuse, ca- } \\
\text { nal de calibre } \\
\text { uniforme } \\
\text { Embouchure inter } \\
\text { ne de la bursa } \\
\text { non chitineuse } \\
\text { et spermathæque } \\
\text { invisible. }\end{array}$ & & $\begin{array}{l}\text { Canal très étrolt } \\
\text { sans dilatations } \\
\text { ni vestibule et } \\
\text { dépourvu de strue } \\
\text { ture chitineuse } \\
\text { autour de son ori } \\
\text { fice interne,s'ou } \\
\text { vrant directement } \\
\text { au scmmet d'une } \\
\text { petite papille } \\
\text { saillante. } \\
\text { Spermatheque inv1 } \\
\text { sible }\end{array}$ \\
\hline Epigynium & & cintre & & $\begin{array}{l}\text { Beaucoup moins } \\
\text { courbe que D. } \\
\text { Pteronyssinus }\end{array}$ & & $\begin{array}{l}\text { Relativement peu } \\
\text { courbe }\end{array}$ \\
\hline $\begin{array}{l}\text { Longueur rela- } \\
\text { tive des pattes } \\
\text { III et IV }\end{array}$ & $\begin{array}{l}\text { P.III une fois } \\
\text { et demie plus } \\
\text { longue que } \\
\text { P.IV }\end{array}$ & $\begin{array}{l}\text { P.III legèrement } \\
\text { pius longue que } \\
\text { P.IV }\end{array}$ & $\begin{array}{l}\text { P.III une fois } \\
\text { et demie plus } \\
\text { longue que } \\
\text { P.IV }\end{array}$ & $\begin{array}{l}\text { P.III plus cour- } \\
\text { te que P.IV }\end{array}$ & $\begin{array}{l}\text { P.III deux fols } \\
\text { plus longue } \\
\text { que P.IV }\end{array}$ & $\begin{array}{l}\text { P,III beaucoup } \\
\text { pius longue que } \\
\text { P.IV }\end{array}$ \\
\hline $\begin{array}{l}\text { Epaisseur rela- } \\
\text { tive des pat- } \\
\text { tes III et IV }\end{array}$ & $\begin{array}{l}\text { P.III plus } \\
\text { épaisse que } \\
\text { P.IV }\end{array}$ & $\begin{array}{l}\text { P. III d'égale } \\
\text { épafsseur a } \\
\text { P.IV }\end{array}$ & $\begin{array}{l}\text { P.III plus } \\
\text { Gpaisse que } \\
\text { P.IV }\end{array}$ & $\begin{array}{l}\text { P.III d'égale } \\
\text { epaisseur a } \\
\text { P.IV }\end{array}$ & $\begin{array}{l}\text { P.III plus } \\
\text { epaisse que } \\
\text { P.IV }\end{array}$ & $\begin{array}{l}\text { P.III plus épaisse } \\
\text { que P.IV }\end{array}$ \\
\hline $\begin{array}{l}\text { Epaisseur re- } \\
\text { lative des } \\
\text { pattes I et II } \\
\text { chez le male }\end{array}$ & $\begin{array}{l}\text { P.I et P.II } \\
\text { d'égale } \\
\text { épaisseur }\end{array}$ & & $\begin{array}{l}\text { P.I beaucoup } \\
\text { pius Epaisse } \\
\text { que P.II }\end{array}$ & & $\begin{array}{l}\text { P.I d'égale } \\
\text { épaisseur a } \\
\text { P.II }\end{array}$ & \\
\hline $\begin{array}{l}\text { Tarses chez le } \\
\text { male }\end{array}$ & $\begin{array}{l}\text { Tarse I avec } \\
\text { un seul ongle } \\
\text { apical. I sans } \\
\text { Tarse II sans } \\
\text { ongle apical. }\end{array}$ & & $\begin{array}{l}\text { TarseIII sans } \\
\text { epines dans sa } \\
\text { partie moyenne }\end{array}$ & & $\begin{array}{l}\text { Tarse III por- } \\
\text { tant } 2 \text { epines } \\
\text { dans sa partie } \\
\text { moyenne }\end{array}$ & \\
\hline $\begin{array}{l}\text { Ecusson hyst6- } \\
\text { rosomal dorsal } \\
\text { chez le male }\end{array}$ & $\begin{array}{l}\text { Plus long que } \\
\text { large }\end{array}$ & & $\begin{array}{l}\text { Plus large que } \\
\text { long }\end{array}$ & & $\begin{array}{l}\text { Plus large que } \\
\text { long }\end{array}$ & \\
\hline $\begin{array}{l}\text { Epimères I } \\
\text { chez le male }\end{array}$ & Séparés & & $\begin{array}{l}\text { Très souvent } \\
\text { contigus ou } \\
\text { fusionnés en } \\
\mathrm{V} \text { ou en } \mathrm{Y}\end{array}$ & & 86́parés & \\
\hline Epistome & Absent & Absent & Absent & Absent & Absent & Absent \\
\hline $\begin{array}{l}\text { Bord posté- } \\
\text { rieur }\end{array}$ & arrond 1 & arrondi & arrond 1 & arrondi & arrondi & arrondi \\
\hline
\end{tabular}




\begin{tabular}{|c|c|c|c|c|c|}
\hline Dermatophagordes & evansi & Euroglyphus & mayne1 & Euroglyphus & Iongior \\
\hline \multirow[t]{2}{*}{$O^{\prime}$} & Q & $\sigma^{\prime}$ & Q & $\sigma^{\prime}$ & Q \\
\hline & $\begin{array}{l}\text { Une partie de } \\
\text { la face dorsale } \\
\text { médiane est str- } \\
\text { 16e longitudi- } \\
\text { nalenent }\end{array}$ & & $\begin{array}{l}\text { P1is cuticulai- } \\
\text { res épais }\end{array}$ & & $\begin{array}{l}\text { Stries et plis } \\
\text { epais plus nom- } \\
\text { breux que chez Eu- } \\
\text { roglyphus maynei }\end{array}$ \\
\hline \multirow[t]{2}{*}{+2} & $\begin{array}{l}\text { Canal de call- } \\
\text { bre non uniforme } \\
\text { Moitis posté- } \\
\text { rieure beaucoup } \\
\text { plus dilatee } \\
\text { que moitie an- } \\
\text { térieure, partie } \\
\text { posterrieure de- } \\
\text { bouche a l'exté- } \\
\text { rieur par un ori- } \\
\text { flce en entonnoir } \\
\text { sans vestibule. } \\
\text { Spermatheque a } \\
\text { paroi chitineuse } \\
\text { beaucoup plus } \\
\text { longue que lar- } \\
\text { ge. }\end{array}$ & & $\begin{array}{l}\text { Embouchure inter- } \\
\text { ne de la bursa, } \\
\text { petite et très } \\
\text { peu sclerifiée. } \\
\text { Papille génitale } \\
\text { ovolde, très } \\
\text { sclérifié, opa- } \\
\text { que et rougeâtre }\end{array}$ & & $\begin{array}{l}\text { S'ouvre a l'exte- } \\
\text { rieur au niveau } \\
\text { d'une formation } \\
\text { ovolde, sclerifiee, } \\
\text { rougestre, et a } \\
\text { l'interieur par } \\
\text { une embouchure } \\
\text { chitinisé }\end{array}$ \\
\hline & $\begin{array}{l}\text { Plus fortement } \\
\text { courbe et plus } \\
\text { long que D. } \\
\text { pteronyssinus }\end{array}$ & & Peu visible & & Peu visible \\
\hline $\begin{array}{l}\text { P.III une fols } \\
\text { et demie plus } \\
\text { longue que P. } \\
\text { IV }\end{array}$ & $\begin{array}{l}\text { P.III légère- } \\
\text { ment plus lon- } \\
\text { gue que P.IV }\end{array}$ & $\begin{array}{l}\text { P.III legere- } \\
\text { ment plus lonf } \\
\text { gue que P.IV }\end{array}$ & $\begin{array}{l}\text { P.III plus court } \\
f \text { que P.IV }\end{array}$ & $\begin{array}{l}\text { P.III légère- } \\
\text { ment plus lon- } \\
\text { gue que P.IV }\end{array}$ & $\begin{array}{l}\text { P.III plus courte } \\
\text { que P.IV }\end{array}$ \\
\hline $\begin{array}{l}\text { P.III plus } \\
\text { Épaisse que } \\
\text { P.IV }\end{array}$ & $\begin{array}{l}\text { P.III d'égale } \\
\text { épaisseur a } \\
\text { P.IV }\end{array}$ & $\begin{array}{l}\text { P.III sensi- } \\
\text { biement d'ega- } \\
\text { le Epaisseur a } \\
\text { P.IV }\end{array}$ & $\begin{array}{l}\text { P.III d'égale } \\
\text { Epalsseur a } \\
\text { P.IV }\end{array}$ & $\begin{array}{l}\text { P.III d'égale } \\
\text { épaisseur a } \\
\text { P.IV }\end{array}$ & $\begin{array}{l}\text { P.III d'egale } \\
\text { epaisseur a } \\
\text { P.IV. Pattes } \\
\text { très fines. }\end{array}$ \\
\hline $\begin{array}{l}\text { P.I d'égale } \\
\text { epaisseur a } \\
\text { P.II }\end{array}$ & : & $\begin{array}{l}\text { P.I d'egale } \\
\text { épaisseur a } \\
\text { P.II }\end{array}$ & & $\begin{array}{l}\text { P.I. d'égale } \\
\text { épaisseur a } \\
\text { P.II }\end{array}$ & \\
\hline \multicolumn{6}{|l|}{$\begin{array}{l}\text { Tarse I et } \\
\text { Tarse II } \\
\text { respectivement } \\
\text { termines par } \\
\text { deux et par un } \\
\text { ongle }\end{array}$} \\
\hline $\begin{array}{l}\text { Plus long que } \\
\text { large et for- } \\
\text { tement retréci } \\
\text { en avant }\end{array}$ & & $\begin{array}{l}\text { ovalaire et bien } \\
\text { développe vers } \\
\text { 1'arrieere du } \\
\text { corps }\end{array}$ & & & \\
\hline Sêparés & & trés écartés & & $\begin{array}{l}\text { très écartés } \\
\text { et courts }\end{array}$ & \\
\hline absent & absent & $\begin{array}{l}\text { bifide mais } \\
\text { peu distinct }\end{array}$ & bifide & $\begin{array}{l}\text { relativement } \\
\text { long. Retréci } \\
\text { fortement vers } \\
\text { 1'avant et } \\
\text { bifide }\end{array}$ & $\begin{array}{l}\text { etroit, long. } \\
\text { et bifide }\end{array}$ \\
\hline arrond 1 & arrond 1 & $\begin{array}{l}\text { aepression } \\
\text { très nette }\end{array}$ & $\begin{array}{l}\text { dépression } \\
\text { légere }\end{array}$ & \begin{tabular}{|l|} 
Partie posté- \\
rieure du corps \\
retrécie et \\
terminée par \\
deux petits \\
lobes
\end{tabular} & $\begin{array}{l}\text { pas de } \\
\text { dépression }\end{array}$ \\
\hline
\end{tabular}


- L'échantillon de poussière doit être examiné de préférence assez rapidement après le prélèvement. Mais pour Cunnington et comme nous l'avons constaté nousmêmes, cette condition n'est pas du tout impérative, l'analyse des poussières pouvant se faire valablement, que celles-ci soient prélevées depuis longtemps ou récemment.

\section{$2^{\circ}$ Méthode}

Notre technique actuelle procède du principe de la méthode de Fain, méthode que nous utilisions au départ, et à laquelle nous avons apporté depuis différentes modifications [23].

Notre façon d'opérer est la suivante :

Utiliser un cylindre en verre gradué (éprouvette) de $100 \mathrm{ml}$, ayant une hauteur de $12 \mathrm{~cm}$ et un diamètre de $3 \mathrm{~cm}$, fermé par un bouchon en verre rodé. Introduire l'échantillon de poussière dans l'éprouvette à l'aide d'un entonnoir de verre. Pour une quantité de poussière égale à une pointe de couteau, environ, verser dans l'éprouvette $60 \mathrm{ml}$ de solution saturée de $\mathrm{CINa}$. ( $\mathrm{Si}$ la quantité de poussière est légèrement plus importante, verser $80 \mathrm{ml}$ de solution saturée de $\mathrm{CINa}$ ). Il est important d'avoir une poussière très diluée dans la solution, de façon à bien distinguer les Acariens flottant en surface. Rincer les bords de l'entonnoir avec une pissette contenant de la solution saturée de $\mathrm{CINa}$ pour entraîner la poussière restante.

Les échantillons de poussière peuvent se présenter sous des aspects différents: une poussière très sèche et poudreuse sera entraînée facilement à l'intérieur de l'éprouvette et son examen ne présente aucune difficulté. Souvent, les échantillons de poussière de literie sont composés de débris laineux et floconneux dans lesquels se trouvent les Acariens. Ces flocons de poussière devront être placés dans l'entonnoir à l'aide d'une pince et dilacérés au moyen d'une aiguille montée, la pince tenant le flocon de poussière, afin de faire tomber quelques Acariens dans l'éprouvette. On opérera de la même façon avec une poussière humide, grasse et compacte.

Une fois la poussière en solution dans l'éprouvette, ajouter 5 à 10 gouttes d'une solution détergente à $4 \%$ ( «Germousse » ou «Teepol »). Fermer l'éprouvette, agiter avec douceur en renversant plusieurs fois l'éprouvette selon un angle de $180^{\circ}$, afin de ne pas abîmer les Acariens. Laisser reposer la solution cinq minutes. Le détergent facilite la sédimentation des poussières et les Acariens flotteront à la surface, portés par la forte densité de la solution saturée de CINa.

Verser la partie supérieure de la solution ( $20 \mathrm{ml}$ environ) dans une boîte de Pétri. Rechercher les Acariens à la loupe binoculaire, grossissement 25 à 40 fois, avec un éclairage latéral bien réglé, de façon à ce que la lumière éclaire tangentiellement la surface du liquide. Régler la loupe sur les éléments flottants (et non sur ceux qui sont sédimentés au fond de la boîte de Pétri). Les Acariens se repèrent assez facilement avec un peu d'habitude, et d'autant mieux que la poussière est suffisamment diluée, car, malgré la sédimentation, il y a toujours des particules de poussière en surface.

Les Acariens apparaissent immobiles, transparents ou argentés, réfringents. 
Pour isoler l'Acarien, après avoir essayé plusieurs systèmes, nous avons trouvé que le plus simple était de le recueillir avec une aiguille montée sur laquelle il adhère facilement. Ensuite, placer une goutte d'eau (6) sur une lame; détacher l'Acarien de l'aiguille montée en roulant celle-ci dans la goutte d'eau (qui ne doit pas être trop importante, car on courrait le risque de perdre l'Acarien au moment où l'on place la lamelle sur la goutte).

Celui-ci se voit d'ailleurs à l'œil nu et l'on peut déjà le repérer entre lame et lamelle. Regarder au microscope, grossissement 100 fois, pour identification.

Cette méthode est en définitive très simple et relativement rapide. Une recherche positive peut se faire en dix minutes, ne dépassant jamais vingt minutes. Un résultat négatif est évidemment beaucoup plus long à rendre, car un échantillon doit être examiné longtemps (une demi-heure environ) avant de conclure à sa négativité. De plus, cette méthode a le grand avantage de pouvoir se faire sur des échantillons de poussière extrêmement réduits (7). Notons également qu'avec cette technique nous avons pu observer des Acariens Pyroglyphides vivants.

\section{$3^{\circ}$ Résultats de nos recherches personnelles}

Sur une période d'étude qui s'étend du $1^{\text {er }}$ avril 1970 au $1^{\text {er }}$ novembre 1971 , nous avons pratiqué 253 examens.

Sur ce total, 233 concernent des poussières de maison prélevées en plaine et 20 des poussières de maison prélevées en altitude.

Nous analyserons distinctement ces deux groupes de résultats.

\section{1) EN PLAine.}

Sur les 233 examens pratiqués, 37 ne pouvaient être interprétés valablement en raison du fait que l'échantillon était en quantité insuffisante $(8) ; 38$ ont été négatifs et 158 ont été positifs.

Ces 158 résultats positifs se répartissent ainsi :

- Acariens Pyroglyphides: 154 :

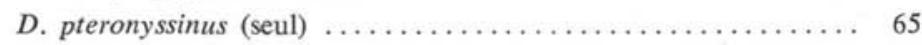

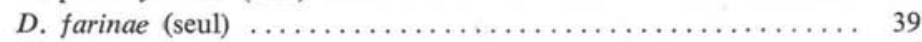

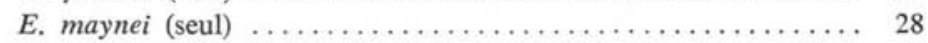

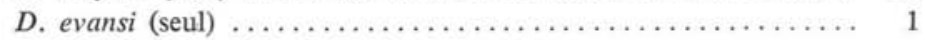

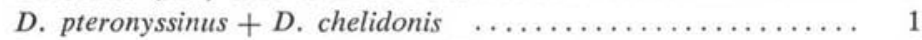

D. pteronyssinus + D. farinae + E. maynei $\ldots \ldots \ldots \ldots \ldots \ldots \ldots \ldots$

(6) On peut aussi utiliser une goutte de solution d'acide lactique à $90 \%$.

(7) La méthode de Fain que nous avons largement détaillée dans une publication antérieure et que nous utilisions au début de nos recherches, nous a donné satisfaction; mais elle exige 1 à $3 \mathrm{~g}$ de poussière, aussi nous avons dû la modifier pour l'adapter aux nombreux échantillons très réduits que nous recevions.

Notons que la méthode de Spieksma exige $5 \mathrm{~g}$ de poussière et celle de Cunnington 50 à $300 \mathrm{mg}$.

(8) Il est à noter qu'avec notre technique actuelle, nous ne rencontrons presque plus d'échantillons insuffisants. 
D. pteronyssinus + D. farinae + (Cheyletus $) \quad \ldots \ldots \ldots \ldots \ldots \ldots . \ldots \ldots$

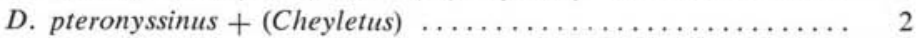

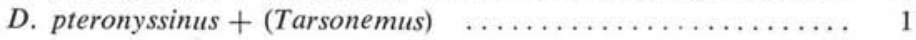

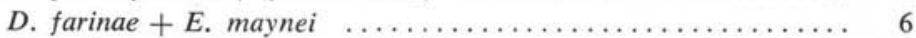

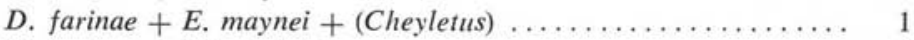

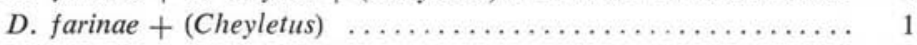

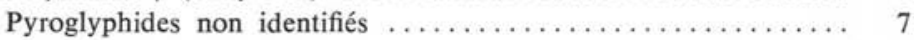

- Autres Acariens : 4 (+6 associés aux Pyroglyphides) :

Cheyletus (seul) $\ldots \ldots \ldots \ldots \ldots \ldots \ldots \ldots \ldots \ldots \ldots \ldots \ldots \ldots \ldots \ldots$ assooiés $)$

Tarsonemus (seul) .................... 2 (+1 associé)

Au total, si pour chacune des espèces nous tenons compte des résultats où elles sont trouvées seules et de ceux où elles sont associées, nous constatons que nous avons vu 71 fois $D$. pteronyssinus, 49 fois $D$. farinae, 36 fois E. maynei, 1 fois $D$. chelidonis, 1 fois D. evansi, 7 fois Cheyletus et 3 fois Tarsonemus.

Il nous est apparu, par ailleurs, qu'une fréquence plus grande des prélèvements positifs était observée en septembre et octobre.

\section{2) EN altitude.}

Récemment, nous avons entrepris la recherche d'Acariens Pyroglyphides dans les régions situées en altitude. Les échantillons de poussière de maison qui nous ont jusqu'à présent été adressés proviennent de Briançon (alt. $1.326 \mathrm{~m}$ ) et de Monetier-lesBains (alt. $1.470 \mathrm{~m}$ ).

Sur les 20 examens que nous avons pratiqués, 13 ont été négatifs et 7 ont été positifs.

Ces 7 résultats positifs se répartissent ainsi :

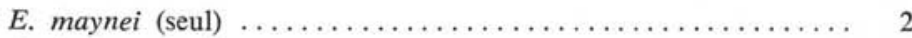

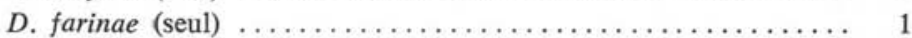

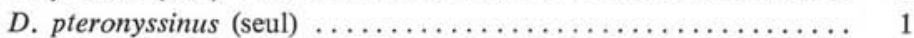

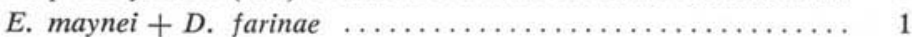

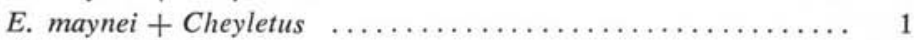

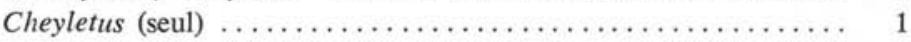

Cette série est trop courte pour conclure. Néanmoins, nous constatons que nous trouvons des Acariens Pyroglyphides en altitude, mais beaucoup moins fréquemment qu'en plaine.

\section{V. - Technique de culture}

La culture des Acariens Pyroglyphides et, en particulier, des deux espèces principales, $D$. pteronyssinus et $D$. farinae, est difficile à réaliser.

Les auteurs étrangers l'ont toutefois réussie pour les deux espèces depuis long- 
temps et ont d'ailleurs constaté que la culture de $D$. pteronyssinus est beaucoup plus difficile à obtenir que celle de $D$. farinae.

En ce qui nous concerne, nous venons d'avoir des résultats satisfaisants dans la culture de ces deux espèces.

Nous avons été aidés par les travaux étrangers, quoique peu prolixes de détails dans ce domaine, et avons procédé de la manière suivante:

\section{$1^{\circ}$ Culture de D. pteronyssinus}

- Les ensemencements de nos milieux ont été fait à partir de poussières de literie positives dans lesquelles nous avions isolé des D. pteronyssinus vivants. (Notons que les échantillons les plus riches sont ceux de la fin de l'été).

- Nos milieux sont faits de squames cutanées humaines (9) additionnées de levure. Pour ce qui est des squames, nous utilisons des poils de barbe provenant de rasoirs électriques, ou mieux, des pellicules du cuir chevelu. Les poils et les pellicules cutanées (10) sont lavés dans de l'acétone. Après lavage, on écarte les poils, on filtre la solution d'acétone sur un tamis de $0,177 \mathrm{~mm}$, on laisse évaporer l'acétone et on récupère la croûte séchée de pellicule et on la broie au mortier. On ajoute à cette croûte pulvérisée un volume égal de poudre de levure «Difco (mais n'importe quelle levure peut convenir). Ce mélange est placé dans une boîte de Pétri et ensemencé avec la poussière positive. On enduit le bord intérieur de la boîte de Pétri avec de la vaseline pour empêcher la fuite des Acariens.

- Nos milieux ainsi constitués sont maintenus en étuve à une température constante de $25^{\circ} \mathrm{C}$ et à un taux permanent d'humidité relative de $75 \%$. Il n'est nul besoin pour cela d'étuve spéciale, les $75 \%$ d'humidité étant obtenus grâce à un petit dessicateur au fond duquel est placée une solution saturée de sel de cuisine. (Ce sel engendre, en effet, une humidité permanente de $75 \%$ à la température de $25^{\circ} \mathrm{C}$ ). Dans ce cas, la boîte de Pétri placée dans le dessicateur ne sera pas fermée.

- Il est à noter que les champignons peuvent être utiles (certains Aspergillus) ou nuisibles. A $80 \%$ d'humidité et au-delà, les champignons poussent trop facilement et risquent de tuer les Acariens. Il est donc préférable de ne pas dépasser $75 \%$, et pour cela, utiliser le sel de cuisine.

- Après un délai de plusieurs semaines nécessaire pour que la culture puisse pousser, nous observons les $D$. pteronyssinus à la loupe binoculaire au grossissement 25 à 40 fois. Ils sont quelquefois difficiles à voir, car ils se cachent sous les différents constituants du milieu que l'on voit bouger ; mais, avec un peu de patience ou en déplaçant délicatement les composants du milieu de culture, on verra très bien les $D$. ptero-

(9) De petites quantités de squames, de l'ordre par exemple de $180 \mathrm{mg}$ sont suffisantes selon Spieksma pour entretenir la totalité d'une culture et produire en deux à trois mois des milliers de D. pteronyssinus. La population peut être maintenue au-delà de 6 mois sur cette quantité de nourriture [5].

(10) Tout récemment, il semble que nous ayons eu aussi des bons résultats avec des pellicules non traitées. 
nyssinus sortir de leur cachette; ils se déplacent lentement, mais avec assurance. Ils ont un aspect légèrement transparent avec des reflets jaunes.

\section{$2^{\circ}$ Culture de $D$. farinae}

- Les ensemencements de nos milieux ont été fait à partir de poussières de literie positives dans lesquelles nous avions isolé des $D$. farinae vivants.

- Pour ces ensemencements, nous avons utilisé la même méthode que pour D. pteronyssinus, avec la seule différence que nous avons maintenu la température du milieu à $20^{\circ} \mathrm{C}(11)$ et non à $25^{\circ} \mathrm{C}$.

- Il faut savoir, comme l'indique la littérature et ainsi que nous l'avons nousmêmes expérimenté, que $D$. farinae peut également se cultiver sur de la farine de seigle, sur des daphnies séchées, sur de la nourriture pour poissons ou sur de la nourriture pour chiens ( Gaine's dogmeal»).

- A l'examen de la culture, les $D$. farinae ressemblent beaucoup aux $D$. pteronyssinus, mais ils sont plus mobiles que ces derniers.

En conclusion, les squames cutanées humaines additionnées de levure en poudre constituent le seul moyen de cultiver D. pteronyssinus (ainsi que E. maynei) et le meilleur moyen de cultiver $D$. farinae. De plus, pour Spieksma, un tel milieu est préférable à tout autre, car la quantité d'antigènes dus à la présence de squames humaines est si faible, tout au moins dans les tests à haute dilution, qu'ils ne contribuent guère à la réaction cutanée observée. En outre, lorsqu'on injecte ces extraits, on n'expose plus le patient à de nouveaux allergènes comme c'est le cas avec les préparations classiques.

\section{VI. - Biologie et écologie}

- Les Pyroglyphides présentent cinq stades dans leur développement:

- œuf,

- larve,

- protonymphe,

- tritonymphe,

- adulte.

- Seul le cycle biologique de $D$. pteronyssinus et de $D$. farinae est jusqu'à ce jour bien connu.

Nous résumons ici les données actuelles de la littérature à ce sujet $[5,37]$ :

L'accouplement, qui a lieu une ou deux fois durant la vie adulte, est suivi de la

(11) Nous avons suivi en cela les conseils de Cunnington. Toutefois, il faut noter que Oshima et Sugita préconisent une température nettement plus élevée, de l'ordre de $30^{\circ} \mathrm{C}$. 
production par la femelle de 20 à 40 œufs environ. [La période de ponte (12) dure plus longtemps chez $D$. farinae que chez $D$. pteronyssinus, mais chez ce dernier le rythme de ponte est plus accéléré que chez le premier, ce qui fait que le nombre d'œufs émis est à peu près identique pour les deux espèces]. L'éclosion de chacun de ces œufs, qui se fait au $6^{\circ}$ jour après la ponte, donnera lieu à la formation d'une larve hexapode, qui après une mue, évoluera en une nymphe octopode (protonymphe, puis tritonymphe) (13), laquelle après une dernière mue deviendra un adulte capable un à trois jours après de se reproduire. Le mâle serait fertile durant la totalité de sa vie, la femelle durant seulement la première moitié de celle-ci. L'évolution de l'œuf à l'adulte dure environ 25 jours (23 à 30 jours). La reproduction est donc sexuée; la parthénogenèse semble inconnue, mais la viviparité serait possible vu que des larves pleinement développées furent observées dans des œufs à l'intérieur du corps de femelles montées, aussi bien chez $D$. farinae que chez $D$. pteronyssinus.

- L'adulte vit en moyenne deux à trois mois et demi [37].

- D. pteronyssinus et $D$. farinae marchent lentement, mais avec assurance. Ils peuvent aller plus vite sans pour autant changer leur direction toutes les fois qu'ils sont exposés à une lumière intense ou à la chaleur. En revanche, D. evansi et E. longior présentent un phototropisme négatif [5].

- Les D. pteronyssinus cherchent à se cacher sous leur substrat alimentaire et se réunissent au fond de la boîte de culture lorsque les conditions de température, de lumière et d'humidité sont extrêmes. Les $D$. farinae essaient de s'évader en grand nombre de la boîte où ils sont cultivés, dès que la nourriture vient à manquer [5].

D. farinae est plus mobile que D. pteronyssinus.

- Les Acariens Pyroglyphides des poussières domestiques, et principalement les trois espèces les plus importantes (D. pteronyssinus, D. farinae, E. maynei), se nourrissent de produits de desquamation épidermiques (pellicules, squames, poils et autres déchets cornés) qui se détachent continuellement de la peau humaine (14) et contribuent à former les poussières des lieux habités [5, 13].

La substance cornée de la peau humaine constitue l'aliment principal de ces Acariens, mais accessoirement il est probable que des déchets cornés d'origine animale (15) soient également utilisés ainsi que doivent l'être les champignons qui apparaissent dans les poussières et les grains de pollen qui tombent de l'air ambiant. Il est à noter que $D$. farinae, $E$. maynei et $E$. longior infestent également les aliments entreposés [5].

(12) La première fois, la période de ponte chez $\mathrm{D}$. farinae dure à $25^{\circ} \mathrm{C}$, environ 30 jours avec un rythme de ponte de 0,8 à 1,4 œuf par jour. Chez D. pteronyssinus, elle est de 20 jours avec un rythme de ponte de 1,2 à 2,5 cufs par jour. Quelquefois pour ces deux espèces, une deuxième période et quelquefois même une troisième période de ponte ont lieu, mais les đufs émis sont alors très peu nombreux.

(13) La protonymphe est caractérisée par une paire de ventouse génitale, la tritonymphe par deux paires. Il est à noter qu'il n'y a pas de deutonymphe chez les Pyroglyphides.

(14) La quantité de squames éliminée par jour par un homme adulte est chiffrée entre 0,7 et $1,4 \mathrm{~g}[5]$.

(15) C'est ainsi que ces Acariens ont été rencontrés dans un oreiller de plumes, sur des peaux conservées, sur divers animaux atteints de lésions galeuses et dans des nids d'oiseaux ou de rats. 
- Le parasitisme est rare.

- La poussière de maison d'habitation constitue par excellence le biotope véritable de ces Acariens Pyroglyphides. La présence dans ce milieu de nombreuses formes immatures associées aux formes adultes renforce cette opinion [13] ; celle de $D$. pteronyssinus, $D$. farinae et $E$. maynei a été particulièrement bien étudiée par tous les auteurs. On trouve ces Acariens surtout dans les chambres, et très accessoirement dans les autres pièces. Leur niche écologique, comme l'a montré Cunnington, est le matelas $(16)[9,17]$. Dans quelques milligrammes de poussière de matelas, on en trouve plusieurs milliers (17) $[9,17]$.

Les échantillons de poussière obtenus par brossage de la surface des matelas contiennent beaucoup plus de Dermatophagoüdes que les échantillons provenant d'ailleurs ; ainsi, en Angleterre, Cunnington a constaté que la densité de D. pteronyssinus contenus dans la poussière de matelas est cent fois plus grande que celle de ceux contenus dans la poussière de la salle de séjour. Il a remarqué également qu'à l'exception peut-être des plumes, les matériaux utilisés dans la confection des matelas ne semblent pas influencer la quantité d'Acariens trouvés. Des essais préliminaires avec trois de ces matériaux (coton, barbes de noix de coco, sisal) ont montré que, malgré la présence de stades de développement récents et de quelques vieux subsistants, le D. pteronyssinus est incapable de se développer sur ces matériaux. Cependant, Cunnington signale avoir trouvé moins de Dermatophagoïdes dans les matelas «Dunlopillo ». Cet auteur a encore constaté que, lorsque le matelas est trop vieux, le Dermatophagoïdes va à l'intérieur du matelas.

Toujours selon Cunnington, l'abondance des Acariens Pyroglyphides est la même dans les échantillons de poussière provenant de demeures humides de vieux asthmatiques que dans ceux recueillis dans des habitations modernes équipées du chauffage central et habitées par des sportifs sains [9]. Il a donc été impossible d'établir, selon cet auteur, une relation étroite entre l'humidité des maisons et le nombre d'Acariens présents dans la poussière du matelas. Cette opinion n'est pas partagée par Voorhorst, Spieksma et Varekamp, qui constatent au contraire qu'en Hollande un gramme de poussière domestique provenant de demeures humides contient neuf fois plus d'Acariens que ceux provenant de demeures sèches $[5,37,39]$.

Par ailleurs, Cunnington, qui a particulièrement étudié au Pest infestation Laboratory de Slough (près de Londres) le cycle vital de $D$. pteronyssinus et de $D$. farinae, constate que, pour ces deux espèces, les conditions les plus favorables à leur développement sont une humidité relative de l'ordre de $80 \%$ et une température modérément élevée, de l'ordre de $25^{\circ}$ à $30^{\circ} \mathrm{C}$. (Notons qu'une telle température est très commune dans le microclimat du lit où dort 6 à 10 heures par jour le corps humain dont la température interne est de $37^{\circ} \mathrm{C}$ ).

(16) Le Dermatophagoïdes fut signalé pour la première fois dans la poussière de plancher et dans la poussière de matelas en 1956 à New York par Baker et ses Collaborateurs [5].

(17) C'est l'estimation de Cunnington; Spieksma trouve une densité d'Acariens nettement plus réduite (500 par gramme) [36]. 
D. farinae semble même préférer des températures plus hautes que $25^{\circ} \mathrm{C}$. Oshima et Sugita ont constaté, en effet, que ce dernier pond davantage d'œufs, et ses formes immatures se développent plus rapidement à $30^{\circ} \mathrm{C}$ qu'à $25^{\circ} \mathrm{C}$ [5]. De plus, D. farinae semble développer des mécanismes de protection pour survivre dans les climats secs [5].

Quoi qu'il en soit, aussi bien pour D. pteronyssinus que pour $D$. farinae, le degré le plus bas toléré pour leur développement serait de $15^{\circ} \mathrm{C}$. Pour ce qui est des températures les plus hautes, les Dermatophagoïdes pourraient supporter plus de $30^{\circ} \mathrm{C}$, voire $35^{\circ} \mathrm{C}$, pendant quelques temps, mais si de telles températures se maintenaient, ils auraient du mal à se développer, mais survivraient.

En définitive, pour Cunnington, le Dermatophagö̈des est un hôte obligatoire de toutes les maisons, et c'est le microclimat régnant dans les matelas qui est important pour son développement, microclimat dont les composantes sont la chaleur, l'humidité et la poussière mélangée aux produits de desquamation épidermiques qui se détachent continuellement de la peau humaine et qui servent de nourriture au Dermatophagoïdes ou aux autres Pyroglyphides des poussières.

Cunnington et Grégory ont montré que les Dermatophagoïdes peuvent être projetés dans l'air en faisant le lit, et on comprend qu'ils puissent de la sorte être inhalés, ce dernier fait ayant été prouvé par certaines observations cliniques. Notons que lorsque ce n'est pas l'Acarien lui-même qui est inhalé, ce sont ses excreta ou ses secreta qui le sont.

Les auteurs hollandais Voorhorst et Spieksma ont constaté que le nombre d'Acariens trouvés dans les échantillons de poussière de maison fluctue selon une périodicité saisonnière avec un maximum à la fin de l'été et un minimum à la fin de l'hiver (le nombre d'Acariens est dix fois plus important en automne qu'au printemps, et cela s'expliquerait par des taux plus élevés d'humidité) $[5,30,36,37,39]$. Ce rythme saisonnier paraît beaucoup moins net aux auteurs anglais.

L'altitude est néfaste au Dermatophagoïdes. La rareté des Acariens Pyroglyphides à des altitudes supérieures à 1.500 mètres coïncide avec une baisse nette de l'humidité relative [5] ; Rufli affirme qu'il n'y a pas de Dermatophagoïdes dans la poussière de maison des lieux de cure classique pour l'asthme, d'altitude élevée, tels que, par exemple, Célérina (1.724 m) ou Pontrésina (1.803 m) [27].

Les Acariens Pyroglyphides des poussières domestiques se trouvent dans tous les pays, mais semblent plus abondants dans les régions où le climat est humide et tempéré. Les espèces rencontrées sont, par ailleurs, variables suivant les pays :

- D. pteronyssinus et $D$. farinae sont les deux seules espèces à être cosmopolites; toutefois, D. pteronyssinus est beaucoup plus répandu que $D$. farinae en Europe, et $D$. farinae est beaucoup plus répandu que $D$. pteronyssinus en Amérique du Nord; aussi Wharton appelle-t-il $D$. farinae la «mite américaine », et $D$. pteronyssinus «la mite européenne »;

- D. chelidonis se voit aux U.S.A., en Europe, en Asie (18), en Afrique ;

(18) D. chelidonis est au Japon la deuxième espèce trouvée dans les poussières de maison au point de vue fréquence [18]. D. pteronyssinus est la première, excepté à Nagoya où il y a très peu de $D$. pteronyssinus mais beaucoup de $D$. farinae. 
- D. evansi se voit au Canada, aux U.S.A., en Europe ;

- E. maynei est très répandu en Europe, mais se voit aussi en Asie et en Amérique du Sud ;

- E. longior, que l'on a isolé dans les poussières de plancher de fermes, se voit au Canada, aux U.S.A., en Europe et en Asie ;

- Sturnophagoïdes brasiliensis et Malayoglyphus intermedius se voient tous les deux en Amérique du Sud et en Asie.

Les Acariens Pyroglyphides sont dans tous les continents les plus répandus des Acariens que l'on peut trouver dans les poussières de maison. Ils sont plus répandus que d'autres Acariens pourtant très communs, tels que les Glycyphagus, les Acarus et les Tyrophagus, et l'on ne doit pas les confondre avec ces derniers.

Entre les Acariens Pyroglyphides des poussières domestiques et les autres Acariens également présents dans ces poussières, existent parfois des interrelations; c'est ainsi que plusieurs espèces de Cheyletidae sont connues pour faire des Pyroglyphidae leur proie. En effet, des constatations de laboratoire ont montré que Cheyletus eruditus (fig. 9), qui est souvent associé au Dermatophagoïdes dans les échantillons de poussière de maison, dévore $D$. pteronyssinus, et prospère aux dépens de lui [5].

Comme autres Acariens que l'on peut trouver dans les poussières de maison, citons aussi les Tarsonemus (fig. 10), qui apparaissent surtout quand le matelas est mouillé.

\section{VII. - Rôle pathogène des Acariens des poussières chez l'homme}

\section{$1^{\circ}$ Allergies respiratoires.}

Depuis les travaux de Voorhorst et Spieksma, la plupart des auteurs pensent que certains Acariens Pyroglyphides des poussières de maison jouent un rôle important dans l'étiologie des allergies respiratoires chez l'homme, soit par inhalation des Acariens eux-mêmes, soit par inhalation de leurs produits d'excrétion ou de sécrétion.

Ainsi, dans l'asthme bronchique et les rhinites dus aux poussières, l'agent sensibilisant ou déclenchant ne serait pas la poussière elle-même, mais les Acariens qu'elle renferme (19) ; parmi ceux-ci, trois espèces ont été reconnues allergisantes: $D$. pteronyssinus, D. farinae et E. maynei [5]. Les tests cutanés, comme le montrent les

(19) Il a été démontré chez quelques patients habitant en plaine et présentant un asthme dépendant de leur lieu de domicile que celui-ci était en fait en relation avec la teneur en Acariens de leur literie [27].

Il a été également rapporté que le taux d'allergène dans la poussière présenterait un maximum de juillet à novembre, période pendant laquelle les malades sensibilisés à l'allergène poussière de maison présenteraient précisément le plus de troubles allergiques; or, c'est pendant cette période que les Acariens présentent leur maximum de développement et c'est en septembre-octobre, qu'ils sont les plus abondants dans les poussières $[13,36]$. 
travaux étrangers, confirment la corrélation étroite qui existe dans la sensibilisation aux poussières de maison et à ces Acariens.

En France, J. Charpin et ses collaborateurs ont également étudié cette allergénicité du Dermatophagoïdes en pratiquant, entre le 15 décembre 1969 et le 31 octobre 1971 , une étude comparée du résultat des tests cutanés à la poussière de maison et au D. farinae, chez 1.829 malades examinés pour des manifestations respiratoires à type d'asthme bronchique ou de coryza spasmodique. Ils ont conclu qu'il existait bien une étroite corrélation entre les réponses cutanées obtenues avec le $D$. farinae et la poussière de maison, puisque, parmi les 421 positifs à $D$. farinae qu'ils ont constatés, 363, soit $86,2 \%$ sont positifs à la poussière. Ces auteurs ont également comparé les tests cutanés à la poussière de maison à différents extraits de $D$. farinae et à un extrait de $D$. pteronyssinus. Cette étude leur a permis de penser que l'allergénicité de $D$. farinae et de D. pteronyssinus était identique, rejoignant en cela l'opinion de Voorhorst et Spieksma (20). Miyamoto et ses collaborateurs ont, de leur côté, signalé qu'il existait une antigénicité croisée entre ces deux espèces [19].

Par ailleurs, il a été constaté, lors de tests cutanés effectués au moyen de différents échantillons de poussière, que l'intensité de la réaction allergique produite était proportionnelle au nombre d'Acariens qu'ils contenaient.

Les auteurs hollandais ont pratiqué des tests d'inhalation avec la poussière de maison et ces Pyroglyphides qui leur ont permis de conclure à l'allergénicité de ces Acariens. De plus, des extraits de Pyroglyphides ont été utilisés par Voorhorst dans la désensibilisation de patients allergiques aux poussières de maison. Cet auteur rapporte que sur les 43 patients désensibilisés à l'aide d'extraits de $D$. pteronyssinus, ceux qui avaient auparavant été désensibilisés à l'extrait de poussière domestique réagirent assez mal à la désensibilisation subséquente à l'extrait de $D$. pteronyssinus, alors que ce ne fut pas le cas chez ceux qui n'avaient reçu que l'extrait de $D$. pteronyssinus d'emblée. Voorhorst en conclut que le résultat clinique le plus important de la désensibilisation avec les extraits de poussière ou de D. pteronyssinus fut une diminution de la gravité des réactions cutanées, qui fut de même degré pour l'un et l'autre extrait, et cette similitude d'action lui semble une preuve immunologique définitive du fait que les deux produits sont complètement identiques au point de vue allergologique [35].

Tous ces faits convergents confirmeraient donc que certains Acariens Pyroglyphides (D. pteronyssinus, D. farinae et $E$. maynei) sont les principaux responsables de l'allergie à la poussière de maison, et en Europe $D$. pteronyssinus serait le principal d'entre eux.

\section{$2^{\circ}$ Allergies cutanées.}

Il est probable que, dans la plupart des cas, les Acariens Pyroglyphides des poussières, et en particulier les Dermatophagoïdes, n'ont pas d'action pathogène directe sur la peau, et qu'ils s'installent sur des lésions déjà existantes dues à Sarcoptes scabiei

(20) Spieksma pense en outre que E. maynei produit le même type d'allergène que D. pteronyssinus et D. farinae [39]. 
ou à un dermatophyte, s'associant donc à eux, car attirés par les squames particulièrement abondantes élaborées au niveau des lésions galeuses ou mycosiques.

Toutefois, le rôle pathogène de ces Acariens Pyroglyphides peut exister, et il est probable que leur action s'exerce bien plus par l'intermédiaire des allergènes qu'ils excrètent ou libèrent après leur mort, que par leur présence réelle, et que la lésion qu'ils engendrent soit une dermatite allergique du type des dermatites de contact.

$3^{\circ}$ Des Dermatophagoïdes ont été trouvés dans des urines humaines, dans des crachats de malades souffrant d'asthme, de syndrome de Loeffler ou d'éosinophilie tropicale (21). Nous ne sommes pas sûrs que ces cas représentent des infections réelles.

\section{VIII. - Prophylaxie}

Au point de vue prophylactique, le meilleur moyen, selon Spieksma, de réduire le nombre d'Acariens est le maintien d'une atmosphère sèche dans les maisons. Pour Solomon, il faudrait bien chauffer les pièces et réduire l'humidité à un taux ne dépassant pas $60 \%$ pour tuer les Acariens Pyroglyphides, et en particulier les Dermatophagoïdes. Selon cet auteur, une exposition pendant 24 heures à $40^{\circ} \mathrm{C}$, ou 8 heures à $45^{\circ} \mathrm{C}$, ou 2 heures à $50^{\circ} \mathrm{C}$, ou 10 minutes à $60^{\circ} \mathrm{C}$ suffirait largement pour les détruire [28].

Inversement, Van Bronswijk rapporte que $D$. farinae peut être tué par une exposition de 24 heures à une température de $-18^{\circ} \mathrm{C}$, si bien qu'au Canada et en Amérique du Nord, où une telle température est communément atteinte pendant au moins plusieurs jours en hiver, une exposition au froid des matelas et des oreillers pourrait être une méthode prophylactique efficace.

Par ailleurs, étant donné que les excreta et les Acariens morts sont aussi allergisants que les Acariens vivants, un complet nettoyage avec l'aspirateur s'impose après avoir tué les Acariens. On devra ainsi nettoyer les matelas, les meubles et le plancher, en particulier au niveau des fentes ou des plinthes, car c'est dans ces derniers endroits que se cachent souvent les Acariens, en particulier D. pteronyssinus, lorsque les conditions de vie deviennent extrêmes.

Néanmoins, même si l'on ne peut tuer les Acariens faute de pouvoir réunir toutes ces conditions dont on vient de parler, le nettoyage régulier des matelas et du plancher avec un aspirateur est cependant fort utile. A titre indicatif, le nombre d'Acariens qui sont transportés par l'air quand on fait le lit est huit fois plus petit lorsque le matelas a été préalablement passé à l'aspirateur.

En revanche, le nettoyage avec l'eau et le savon ne réduit pas nécessairement le nombre d'Acariens, car les fentes où ils se réfugient sont rarement mouillées et, en outre, il faut plus de deux jours pour qu'un Dermatophagoïdes se noie dans une solution savonneuse [5].

(21) La découverte d'Acariens des poussières dans les crachats avait déjà été faite dès 1944 par Carter, Wedd et D'Abrera dans l'Ile de Ceylan. Ces auteurs avaient alors soupçonné sans l'expliquer que ces Acariens qu'ils ne connaissaient pas vraiment pouvaient jouer un rôle dans l'étiologie de certaines manifestations pulmonaires fugaces avec éosinophilie sanguine, manifestations qu'ils décrivirent sous le nom d'Acariase Pulmonaire. 


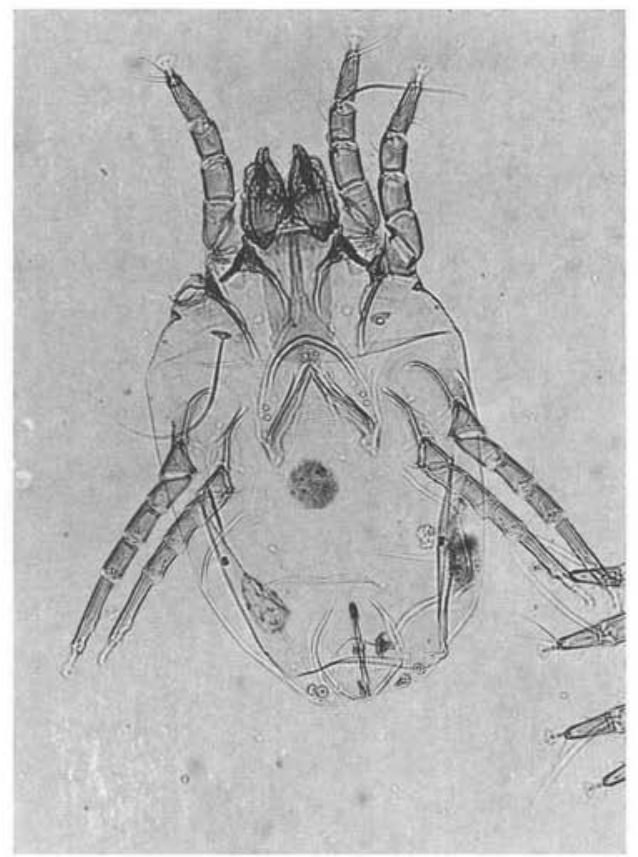

FIG. 1. - Dermatophagoïdes pteronyssinus, Trouessart 1897. Femelle ( $\times$ 100)

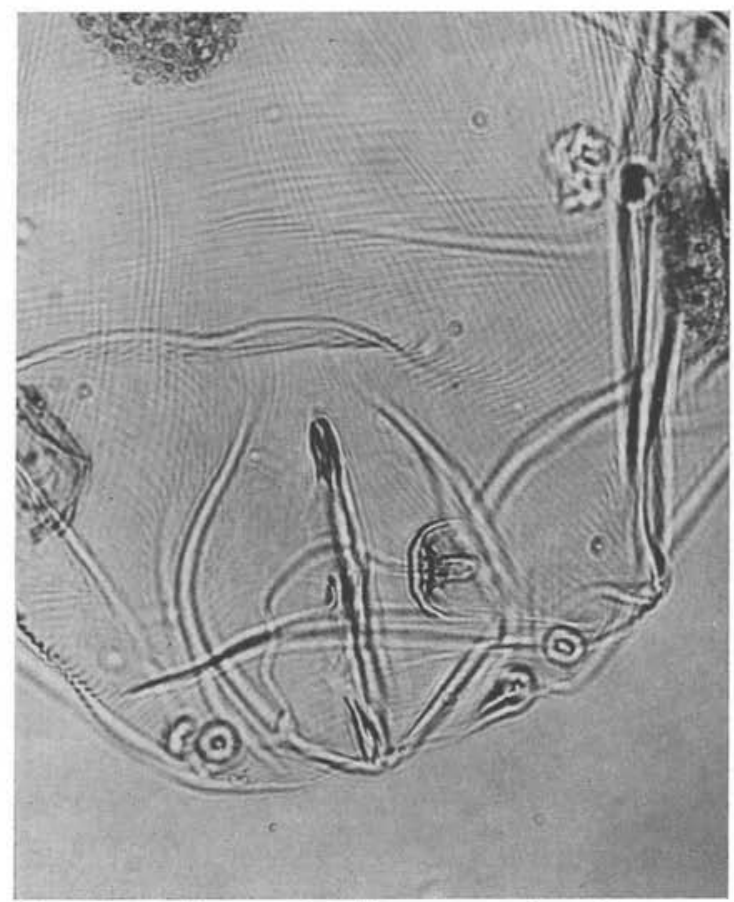

FIg. 2. - Dermatophagoïdes pteronyssinus, Trouessart 1897. Femelle: détail bursa copulatrix ( $\times 400)$ 


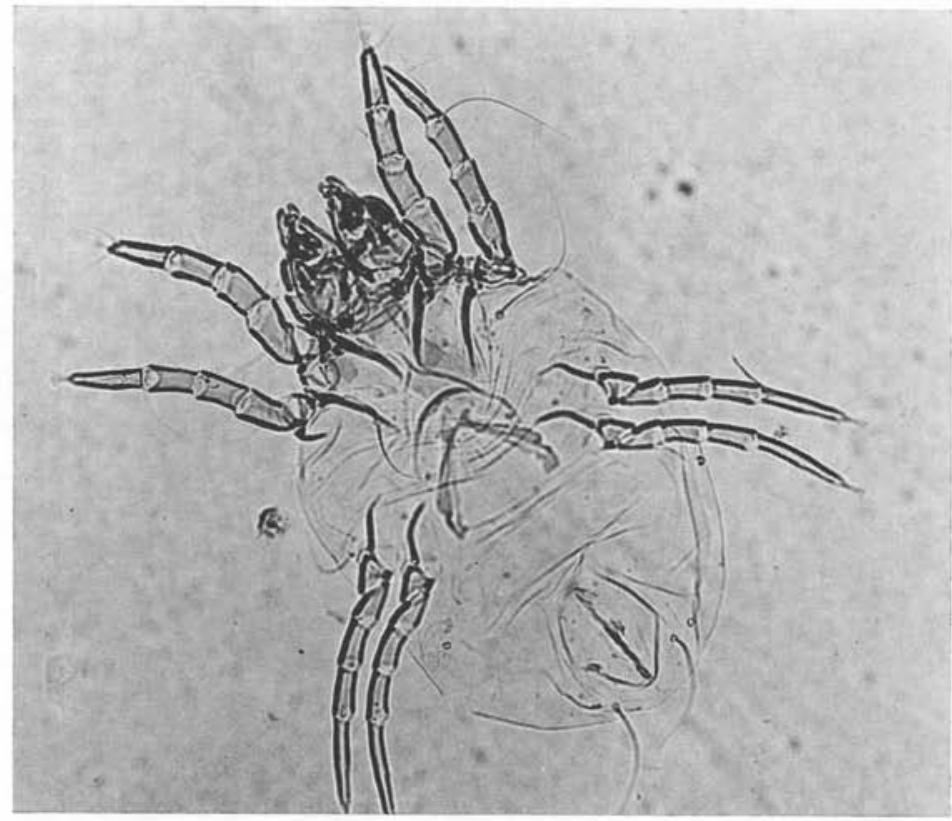

FIG. 3. - Dermatophagoïdes farinae, Hughes 1961. Femelle ( $\times 100)$

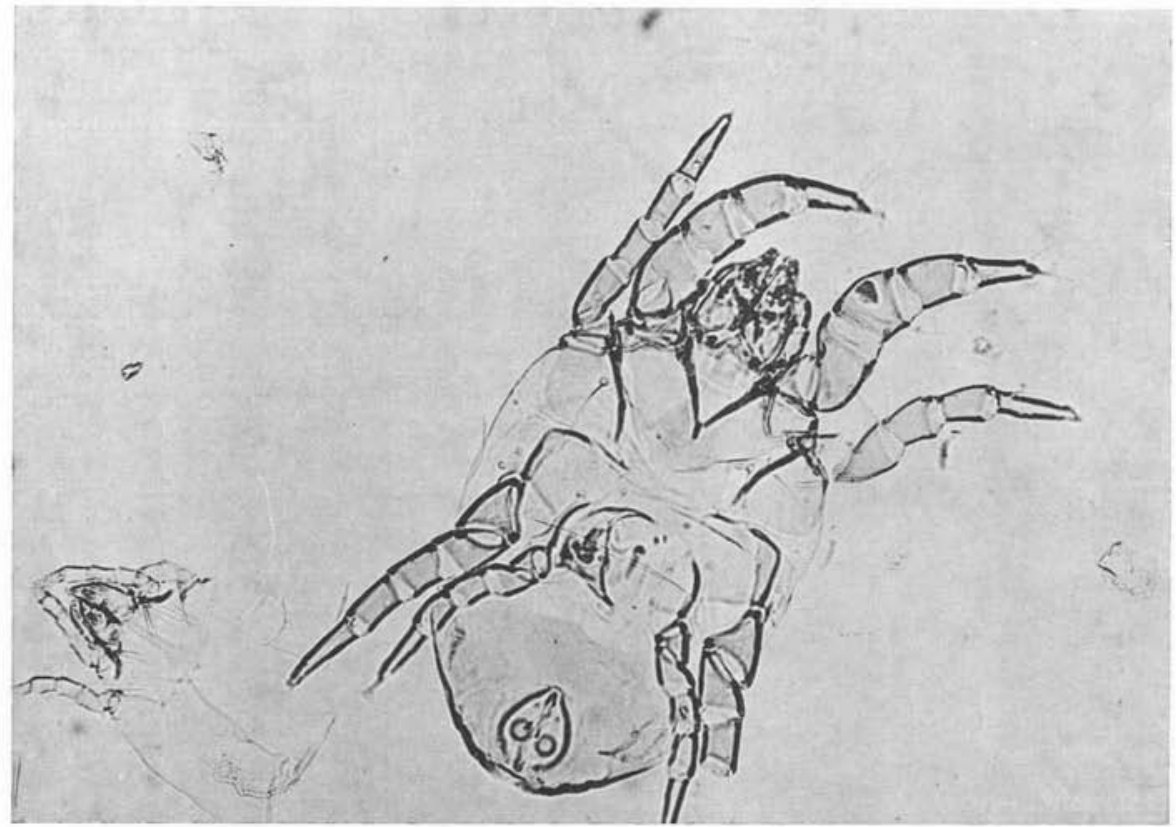

FIG. 4. - Dermatophagö̈des farinae, Hughes 1961. Mâle (× 100) 


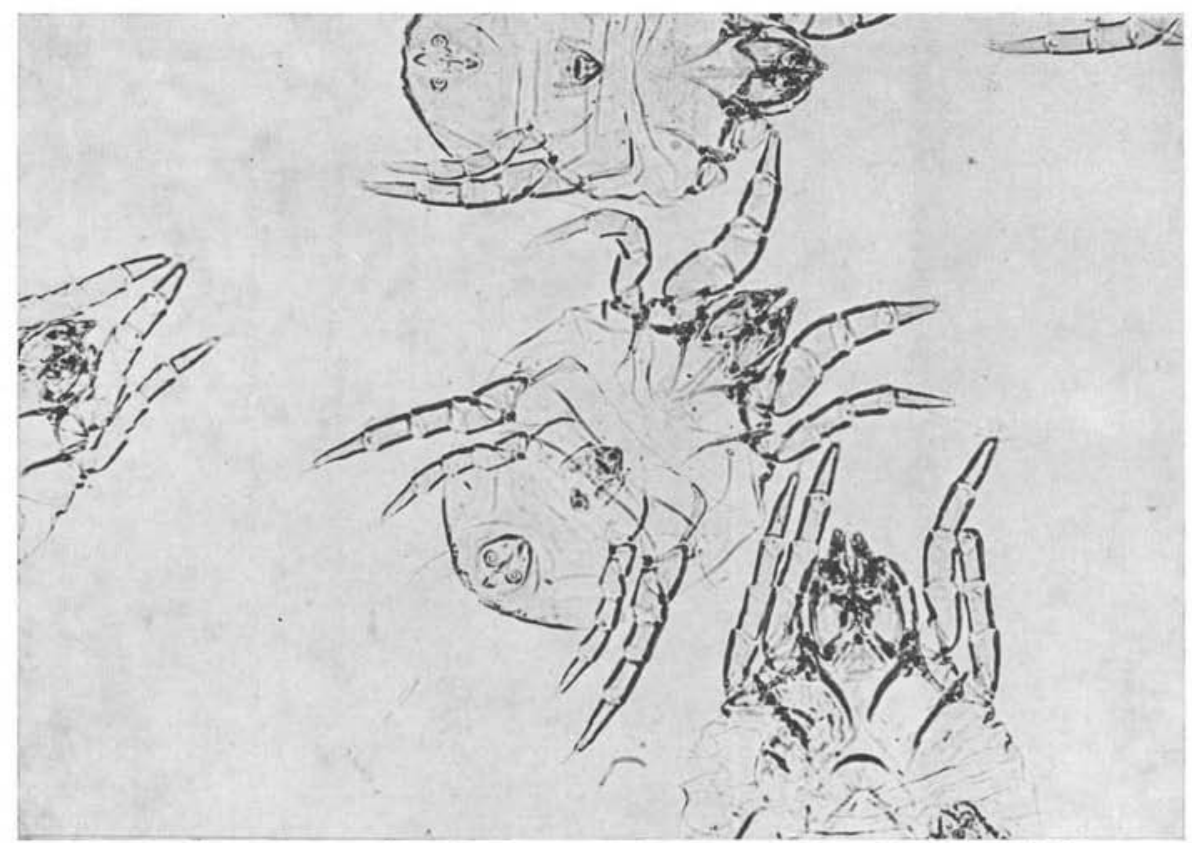

FIG. 5. - Dermatophagoïdes farinae, Hughes 1961. Mâles et Femelle (X 100)

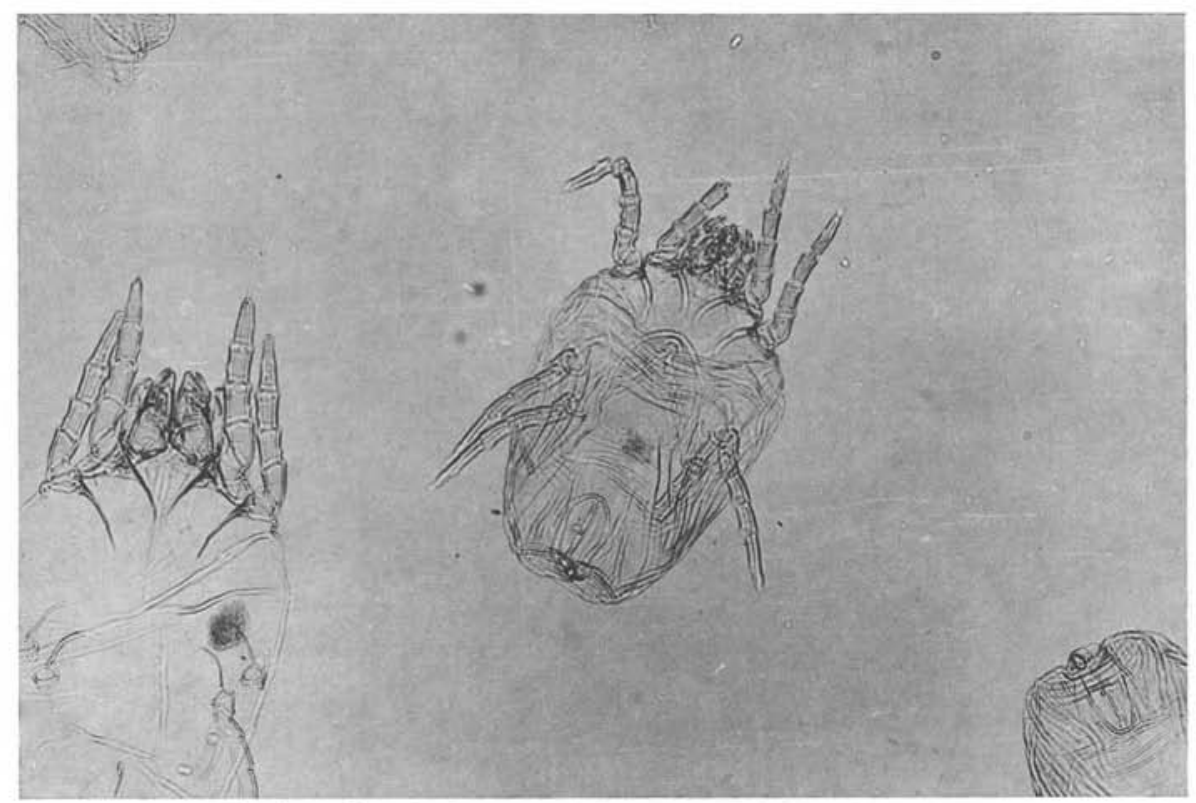

FiG. 6. - Euroglyphus maynei, Cooreman 1950. Femelle $(\times 100)$ 


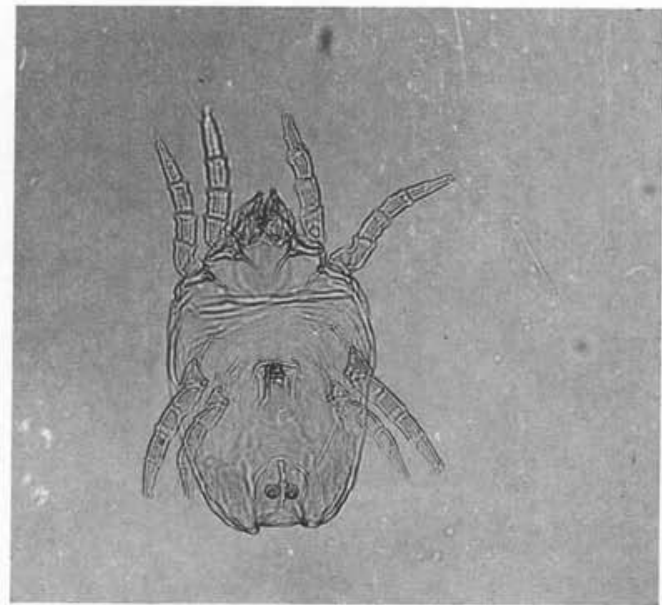

FIG. 7. - Euroglyphus maynei, Cooreman 1950. Mâle (X 100)

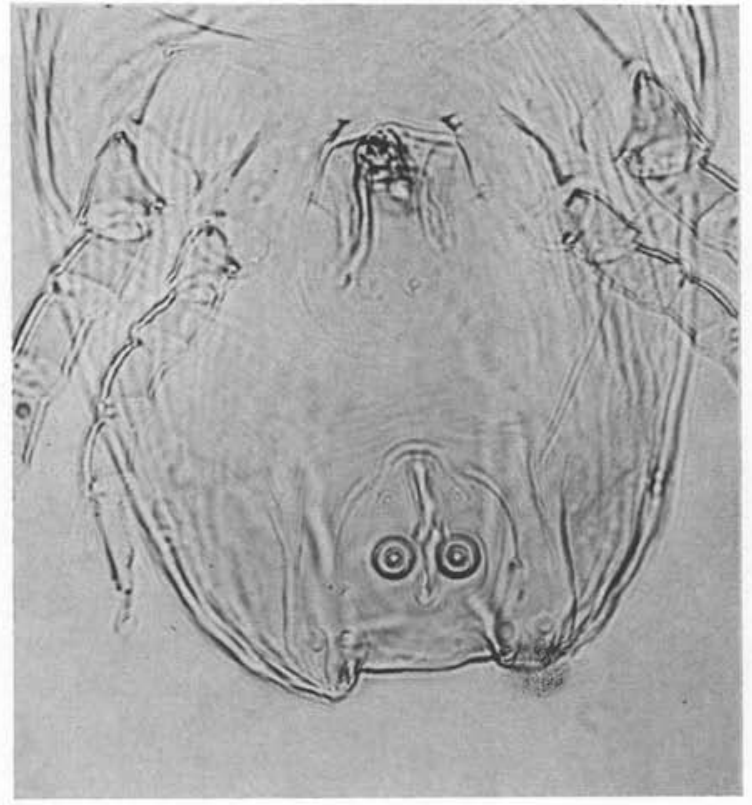

FIG. 8. - Euroglyphus maynei, Cooreman 1950. Mâle : détail bord postérieur $(\times 400)$ 


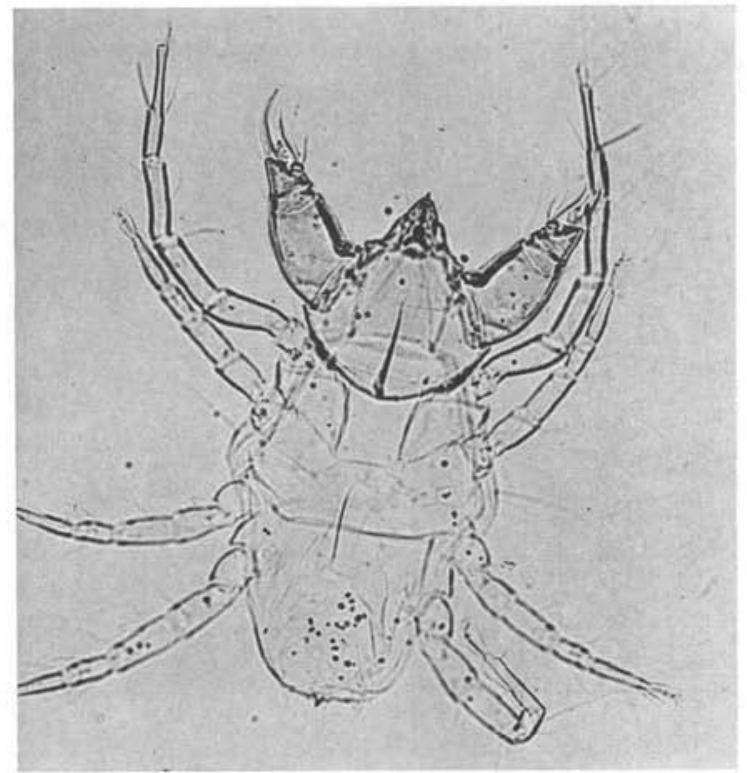

FIG. 9. - Cheyletus eruditus $(\times 100)$

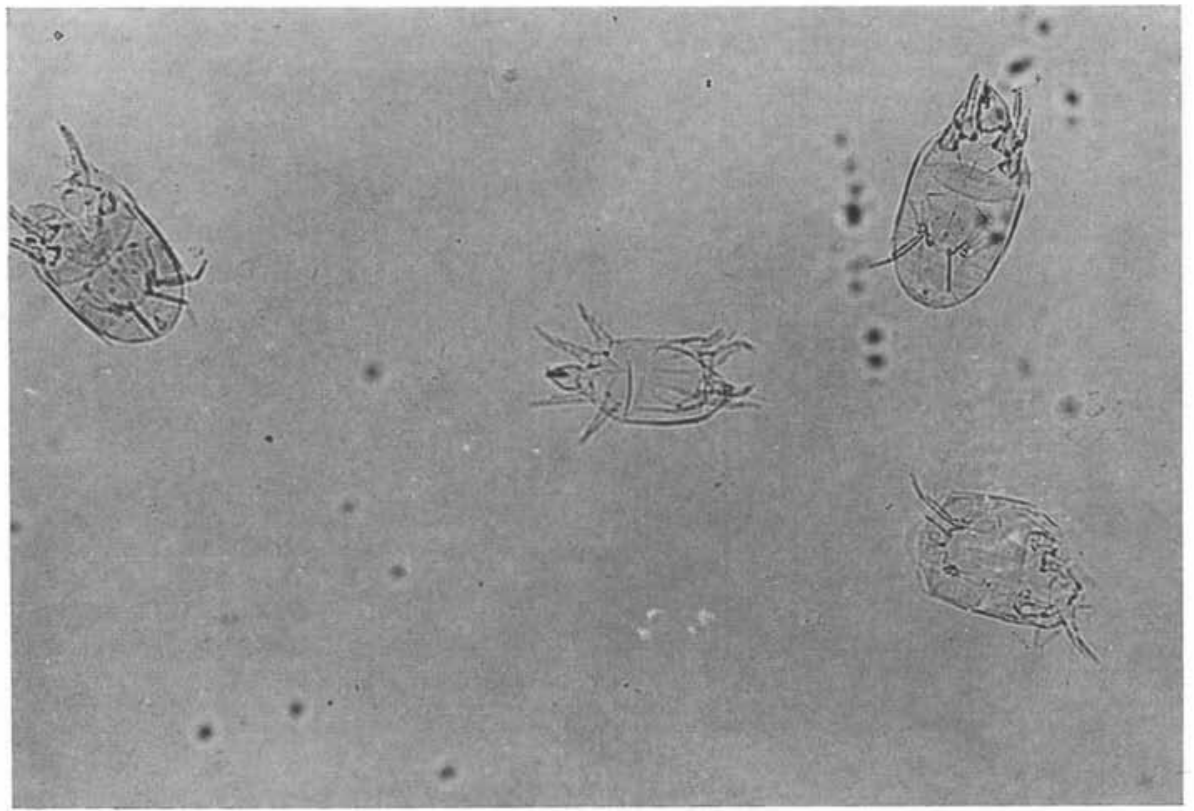

FIG. 10. - Tarsonemus sp. $(\times 100)$ 


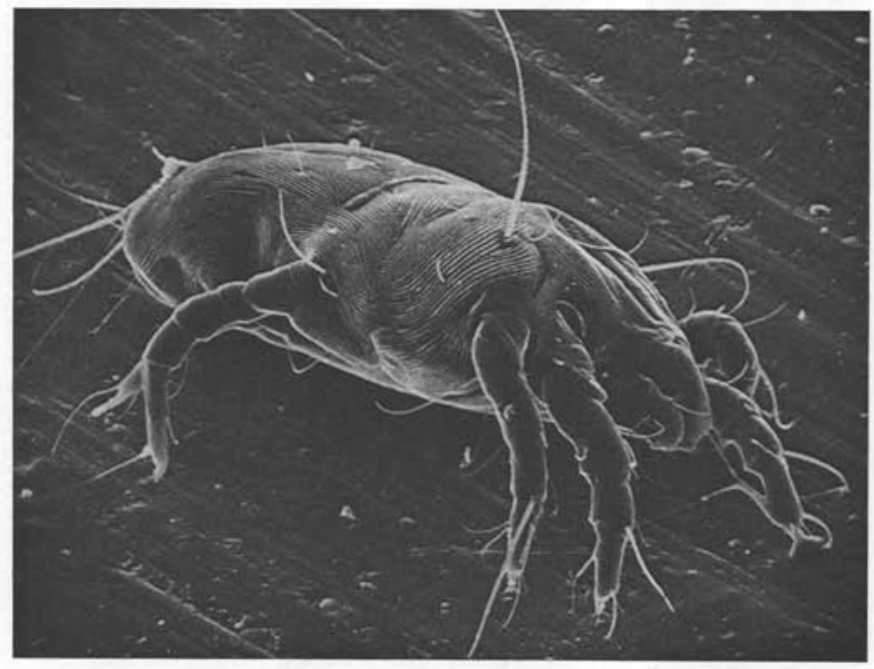

FIG. 11. - Dermatophagoïdes pteronyssinus, Trouessart 1897 (Grossissement 400 ). Photo prise au microscope électronique à balayage (*)

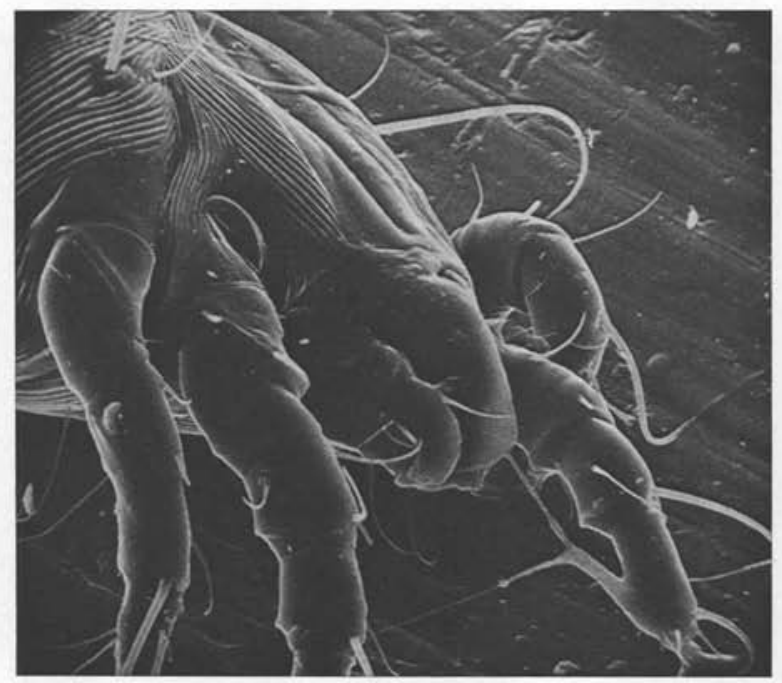

FIG. 12. - Dermatophagoïdes pteronyssinus, Trouessart 1897. Détail : partie antérieure (Grossissement 800). Photo prise au microscope électronique à balayage $(*)$ 


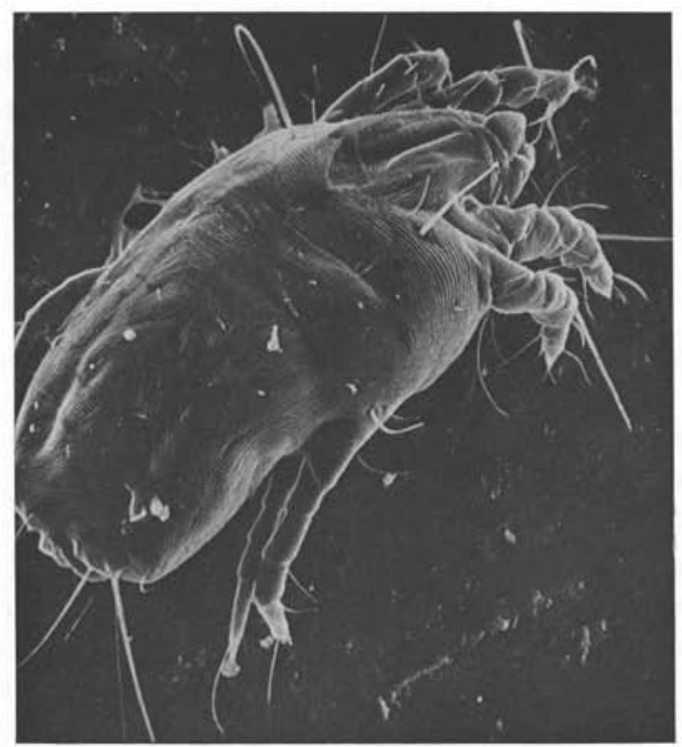

FiG. 13. - Dermatophagoïdes pteronyssinus, Trouessart 1897 (Grossissement 400). Photo prise au microscope électronique à balayage ${ }^{*}$ )

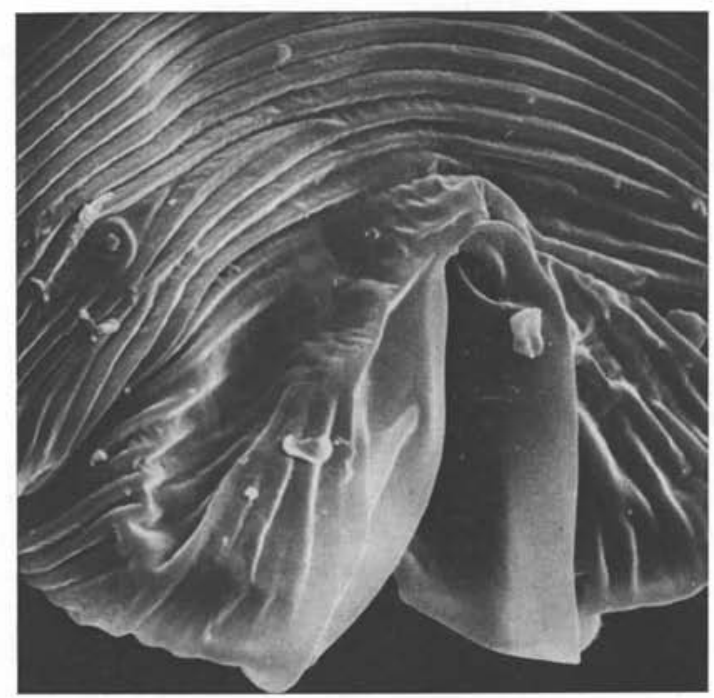

FIG. 14. - Dermatophagoïdes pteronyssinus, Trouessart 1897. Détail : région anale (Grossissement 3000). Photo prise au microscope électronique à balayage $\left({ }^{*}\right)$

(*) Les photos au microscope électronique à balayage ont été réalisées avec le JEOL type JSM. U3 que les établissements JEOL ont mis aimablement à notre disposition. 
Signalons enfin que l'allergène élaboré par ces Acariens Pyroglyphides peut être presque complètement supprimé par un rayonnement aux ultraviolets de deux heures [5]. Quant au D.D.T., il est inefficace contre ces Acariens; le lindane est en revanche actif, mais son emploi dans une maison n'est pas sans danger pour l'homme.

\section{Conclusion}

$1^{\circ} \mathrm{La}$ mise en évidence des Acariens Pyroglyphides dans les échantillons de poussière de maison est techniquement possible à condition que la méthode de recherche soit bien « au point».

$2^{\circ}$ Dans les échantillons de poussière domestique que nous avons analysés et qui proviennent essentiellement du sud-est de la France, c'est le D. pteronyssinus que nous avons trouvé le plus fréquemment.

Mais il n'est pas impossible que cette espèce, qui est également la plus fréquente en Europe et qui serait la principale responsable de l'allergie à la poussière de maison, soit en certains endroits remplacée par d'autres espèces d'Acariens. Aussi, d'autres études portant sur toutes les régions de la France sont nécessaires pour étudier, dans notre pays, la répartition géographique des diverses espèces d'Acariens Pyroglyphides, ainsi que leur rôle respectif dans l'allergie due à la poussière de maison.

$3^{\circ}$ Ces nouvelles données sur l'allergénicité des Acariens Pyroglyphides des poussières de maison sont très importantes par les conséquences qui en découlent.

En effet, dans les allergies respiratoires, on pourrait d'une part concevoir un traitement prophylactique comportant la destruction des Acariens qui auront été identifiés dans les poussières domestiques, et d'autre part envisager de pratiquer une désensibilisation spécifique au moyen d'extraits obtenus à partir de l'élevage de ces mêmes Acariens.

\section{Bibliographie}

1. Araujo (A.), Callot (J.), Basset (A.), 1968. - Rôle des Acariens dans les allergies aux poussières (note préliminaire). Bull. Soc. Fr. Derm. Syph., 75, 696-697.

2. Araujo-Fontaine (A.), Basset (A.), Callot (J.), Maleville (J.), Wagner (M.) et FousSEREAU (J.), 1970. - Anticorps sériques anti-poussière dans l'atopie. Rôle antigénique des Acariens des poussières domestiques. Mise en évidence d'une similitude antigénique entre les poussières domestiques et certaines espèces d'Acariens. C.R. Soc. Biol., 164, 12, 2650-2654.

3. Basset (A.), Maleville (J.), Foussereau (J.), Grosshans (E.), Heid (E.), Schubert (B.) et Fontaine (A.), 1969. - Acquisitions récentes dans l'exploration de l'allergie en dermatologie. Rev. des C.H.U., 8, 63-66.

4. Berrens (L.), Young (E.) et Zuidema (P.), 1971. - A comparative chemical and clinical investigation of house-dust extracts from Alpine and Lowland Régions. Acta Allergologica, 26, 200-212. 
5. BronswiJk Van (J. E. M. H.) et Sinha (R. N.), 1971. - Pyroglyphid mites (Acari) and House-dust Allergy. J. Allerg., vol. 47, $\mathrm{n}^{\circ} 1$, p. 31-51.

6. Charpin (J.), Penaud (A.), Nourrit (J.), Autran (P.) et Razzouk (H.), 1971. - Allergie aux poussières domestiques et Dermatophagoïdes. Communication à la Soc. Fr. d'Allerg. du 14 janvier, Rev. Fr. d'Allerg., 11, 4, 315-328.

7. - Autran (P.), Penaud (A.), Nourrit (J.), et Razzouk (H.), 1972. - Un parasite de la poussière domestique: le Dermatophagoïdes. Rôle dans l'allergie respiratoire. La nouvelle Presse médicale, tome $1, \mathrm{n}^{\circ} 13$, p. 859-862.

8. Cunnington (A. M.) et Gregory (P. H.), 1968. - Mites in Bedroom Air, Nature Lond, 217, 1271-1272.

9. - 1969. - Aspects particuliers de l'allergie aux Acariens de la poussière de maison. Médecine et Hygiène, 8 octobre, $\mathrm{n}^{\circ}$ 887, p. 1139.

10. FAIN (A.), 1964. - Notes sur le genre Dermatophagoïdes Bogdanov. Description d'une espèce nouvelle (Acarina: Psoroptidae). Rev. Zool. Bot. Afr., 69 (1-2) : 201-205.

11. —, 1965. - Les Acariens nidicoles et détriticoles de la famille Pyroglyphidae Cunliffe. Rev. Zool. Bot. Afr., 72 (3-4): 257-288.

12. —, 1966. - Nouvelle description de Dermatophagö̈des pteronyssinus (Trouessart, 1897). Importance de cet Acarien en pathologie humaine (Psoroptidae: Sarcoptiformes). Acarologia, $8: 302-327$.

13. - , 1966. - Allergies respiratoires produites par un Acarien (Dermatophagoïdes pteronyssinus) vivant dans les poussières des habitations. Bull. Acad. Roy. Med. Belgique, $6: 479-499$.

14. —, 1967. - Le genre Dermatophagoïdes Bogdanov 1864, son importance dans les allergies respiratoires et cutanées chez l'homme (Psoroptidae: Sarcoptiformes). Acarologia, 9: 179-225.

15. Frankland (A. W.), Mc Even (L. M.), Feinberg (J. G.), 1970. - Skins Reactions to Dust and Mites. Int. Arch. Allergy, 37 : 351-356.

16. Hughes (A. M.), 1961. - The mites of stored food. London, H.M. Stationery Office (1961), Ministery of Agric., Fish. And Food. Techn. Bull., n 9, 142-152.

17. Maunsell (K.), Wraith (D. G.) et Cunnington (A. M.), 1968. - Mites and house-dust allergy in bronchial asthma. The Lancet, 1267-1270.

18. MiYamoto (T.), Oshima (S.), Ishizaki (T.) et Sato (S.), 1968. - Allergenic identity between the common floor mite (Dermatophagoïdes farinae Hughes, 1961) and house dust as a causative antigen in bronchial asthma. J. Allergy, 42, 14-28.

19. - - - Mizuno (K.), Sasa (M.) et IshizaKi (T.), 1969. - Cross-antigenicity among six species of dust mites and house-dust antigens. J. Allergy, 44, 228-238.

20. - , - et IsHizaKI (T.), 1969. - Antigenic relation between house-dust and a dust mite, Dermatophagoïdes farinae Hughes, 1961 by a fractionation method. J. Allergy, 44, 282-291.

21. -, - Domae (A.), Takahashi (K.), IZeki (M.), Tanaka (T.) et Ishizaki (T.), 1970. Allergic Potency of different house dusts in inoculation to contained mites. Ann. Allergy, 28, 405-412. 
22. Morrison-Smith (J.), Disney (M. E.), Williams (J. D.) et Goels (Z. A.), 1969. - Clinical significance of skin reactions to mite extracts in children with asthma. British Medical journal, 2, 723-726.

23. Nourrit (J.), Penaud (A.), Timon-David (P.), Autran (P.) et Nicoli (R. M.). - Méthode simplifiée pour la recherche des Acariens allergisants des poussières de maison sur des échantillons réduits. Bull. Soc. Path. Exot. (sous presse).

24. Penaud (A.), Nourrit (J.), Timon-David (P.), Autran (P.) et Charpin (J.), 1971. Acariens Pyroglyphides dans les poussières domestiques. Marseille Médical, 5, 363368.

25. Pepys (J.), 1969. - Importance clinique des mites. Médecine et Hygiène, $n^{\circ}$ 887, p. 1138.

26. - Chan (M.) et Hargreave (F. E.), 1968. - Mites and house-dust allergy. Lancet, 1270.

27. Rufli (M.), Rufli (Th.) et Schuppli (R.), 1969. - Etude comparative expérimentale et clinique de l'allergie aux mites. Médecine et Hygiène, $\mathrm{n}^{\circ}$ 887, p. 1139.

28. Solomon (M. E.), 1961. - Mites in houses, shops and other occupied buildings, The 《Sanitarian 》, 69, 291.

29. - et Cunnington (A. M.), 1964. - Rearing acaroid mites. Acarologia, 6, 339.

30. Spieksma (F. Th. M.), 1967. - The house-dust mite Dermatophagoïdes pteronyssinus producer of the house-dust allergen. Thesis Leiden, 1 vol., $65 \mathrm{p}$.

31. - , 1967. - Occurence and properties of the house-dust mite Dermatophagoïdes pteronyssinus. International Rhinology, 5, 162-167.

32. - , et Spieksma-Boezeman (M. I. A.), 1967. - The mite fauna of house-dust with particular reference to the house-dust mite Dermatophagoïdes pteronyssinus (Trouessart, 1897). (Psoroptidae: sarcoptiformes). Acarologia, 9, 226-241.

33. - et Voorhorst (R.), 1969. - Comparison of skin reactions to extracts of house-dust mites, and human skin scales. Acta Allergologica, 24, 124-146.

34. Trinca (J.-C.), Stringer (K.-C.), Drummond (V.-G.) et Briston, 1969. - Rôle d'une mite Dermatophagoïdes pteronyssinus dans le pouvoir allergénique de la poussière de maison. Méd. J. Aust., 1, 1177.

35. Voorhorst (R.), 1970. - Quantitative aspects of the problem of house-dust and housedust mites. Acta Allergologica, 25, 237-254.

36. - - Spieksma-Boezeman (M. I. A.) et Spieksma (F. Th. M.), 1964. - Is a mite (Dermatophagoïdes sp.) the producer of the house-dust allergen? Allergie und Asthma, 10, 329-334.

37. - S Sieksma (F. Th. M.), Varekamp (H.), Leupen (M. J.), et Lyklema (A. W.), 1967. The house-dust mite (Dermatophagoïdes pteronyssinus) and the allergen it producesIdentity with the house-dust allergen. J. Allergy, 39, 325-339.

38. —, et - 1969. - Recent progress in the house-dust mite problem. Acta Allergologica, 24, 115-123.

39. —, - et VareKamp (H.), 1969. - House-dust atopy and the house-dust mite. (Dermatophagoïdes pteronyssinus). Staflen's Scientific Pub Co Leiden, 1 vol., 159 p., 73 figs.

40. Wharton (G. W.),, 1970. - Mites and commercial extracts of house-dust. Science (American association for the advancement of science), 167, 1382-1383.

41. Young (E.) et Bangma (P. J.), 1970. - Mites and house-dust Allergy. Acta Allergologica, $25,25-36$. 